Review

\title{
Structural brain plasticity in adult learning and development
}

\author{
Martin Lövdén ${ }^{\mathrm{a}, \mathrm{b}, *}$, Elisabeth Wenger ${ }^{\mathrm{b}}$, Johan Mårtensson ${ }^{\mathrm{b}, \mathrm{c}}$, Ulman Lindenberger ${ }^{\mathrm{b}}$, Lars Bäckman ${ }^{\mathrm{a}}$ \\ a Aging Research Center, Karolinska Institutet E Stockholm University, Gävlegatan 16, 11330 Stockholm, Sweden \\ b Center for Lifespan Psychology, Max Planck Institute for Human Development, Lentzeallee 94, 14195 Berlin, Germany \\ ${ }^{c}$ Department of Psychology, Lund University, Box 213, 22100 Lund, Sweden
}

\section{A R T I C L E I N F O}

\section{Article history:}

Received 18 October 2012

Received in revised form 4 February 2013

Accepted 19 February 2013

\section{Keywords:}

Experience-dependent plasticity

Brain volume

Structural brain plasticity

Structural brain changes

Learning

Neuroplasticity

Aging

\begin{abstract}
A B S T R A C T
Recent research using magnetic resonance imaging has documented changes in the adult human brain's grey matter structure induced by alterations in experiential demands. We review this research and relate it to models of brain plasticity from related strands of research, such as work on animal models. This allows us to generate recommendations and predictions for future research that may advance the understanding of the function, sequential progression, and microstructural nature of experience-dependent changes in regional brain volumes. Informed by recent evidence on adult age differences in structural brain plasticity, we show how understanding learning-related changes in human brain structure can expand our knowledge about adult development and aging. We hope that this review will promote research on the mechanisms regulating experience-dependent structural plasticity of the adult human brain.
\end{abstract}

(c) 2013 Elsevier Ltd. All rights reserved.

\section{Contents}

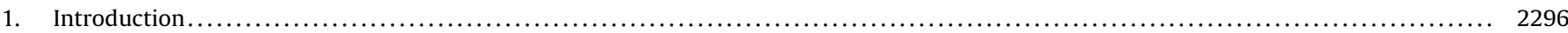

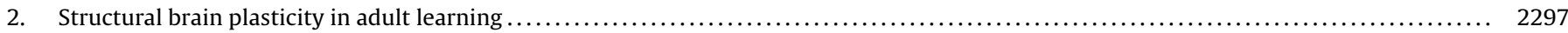

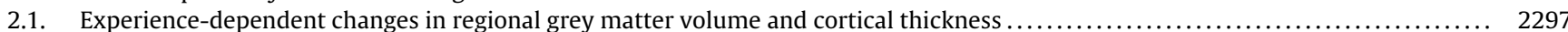

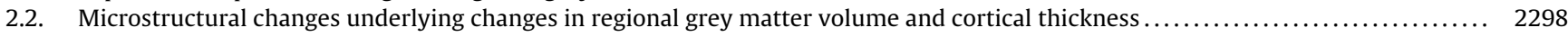

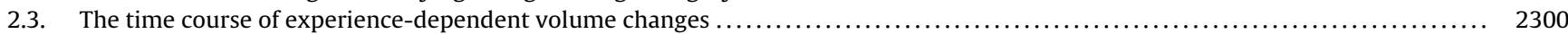

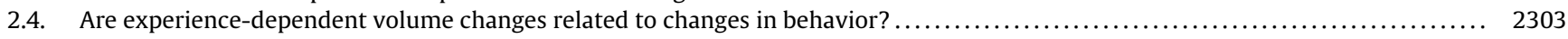

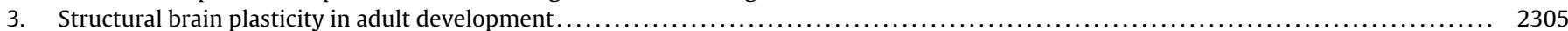

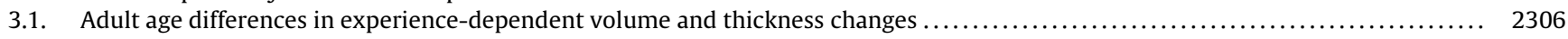

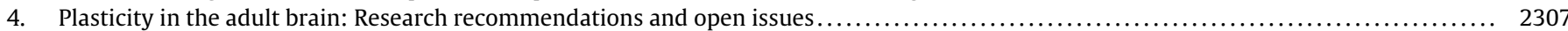

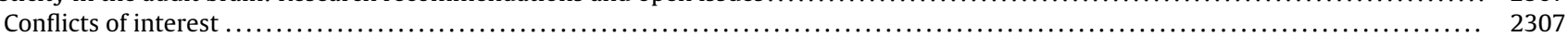

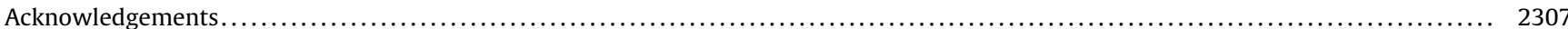

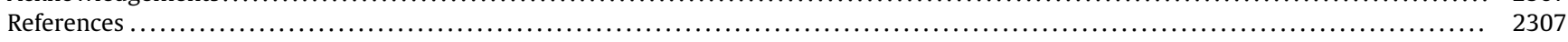

\section{Introduction}

The human brain has a large degree of plasticity, the capacity to adapt to changing demands by altering its structure (Lövdén et al., 2010). Recent research using techniques for imaging the human brain in vivo, particularly magnetic resonance (MR) imaging, suggests that the brain's macrostructure manifests experience-dependent plasticity in adulthood (Draganski et al., 2004, 2006). Although experience-dependent morphological brain

\footnotetext{
* Corresponding author at: Aging Research Center, Karolinska Institutet \& Stockholm University, Gävlegatan 16, 11330 Stockholm, Sweden. Tel.: +46 734428338.

E-mail address: Martin.Lovden@ki.se (M. Lövdén).
}

changes have been investigated in animal models for a long time (Altman and Das, 1964; Greenough et al., 1973; Rosenzweig et al., 1962, 1964; Volkmar and Greenough, 1972), the discovery that such changes are detectable with MR imaging has opened up new avenues for research. In particular, structural correlates of learning can now be identified and studied in humans.

Bidirectional dynamic interactions between brain and behavior at different timescales are at the heart of cognitive lifespan development (e.g., Lindenberger et al., 2006). With the study of experience-dependent anatomical brain changes in humans, the mechanisms underlying these interactions can be examined in greater depth, thereby providing a more mechanistic basis for concepts such as cognitive reserve (Stern, 2009) or brain 
maintenance (Nyberg et al., 2012). Although microstructural brain changes at the cellular level cannot be easily probed currently, imaging of experience-dependent changes in the brain's macrostructure offers a unique window into human learning and development.

Here we review the available evidence on experiencedependent morphological changes in the adult human brain, focusing on alterations in grey matter volume and cortical thickness. Currently, much research in this field is devoted to establishing and extending the basic phenomenon of experience-dependent structural changes. The formulation of explicit hypotheses and concomitant research about the mechanisms involved is relatively sparse. Based on theoretical considerations and empirical work from related strands of research, such as animal models, we generate predictions that may advance understanding of the function, sequential progression, and microstructural nature of volume and thickness changes. The evidence on these predictions is reviewed, and further avenues for testing specific hypotheses are delineated. Finally, we highlight the ways in which understanding experience-related changes in human brain structure will contribute to the understanding of adult development and aging, and review the evidence on adult age differences in structural brain plasticity. With our focus, we hope to extend related recent reviews (May, 2011; Thomas and Baker, in press; Zatorre et al., 2012) by providing a research agenda addressing the mechanisms that regulate changes in experience-dependent structural plasticity of the human brain.

\section{Structural brain plasticity in adult learning}

\subsection{Experience-dependent changes in regional grey matter volume and cortical thickness}

In the early 1960s, Rosenzweig et al. $(1962,1964)$ were the first to apply the complex enrichment paradigm (e.g., Hebb, 1949) to study effects of experience on the brain. This paradigm is also referred to as environmental enrichment and involves housing animals (typically rats or mice) in a cage containing toys that are frequently changed. It should be noted however that the enriched condition is only enriched relative to standard cages housing these strains of animals in isolation, and not relative to what the population of animals that live in the wild experiences. Rosenzweig and colleagues reported changes in brain weight (Rosenzweig et al., 1962, 1964) and cortical thickness (Bennett et al., 1964; Rosenzweig et al., 1972) following environmental enrichment in rats (see Anderson, 2011; Markham and Greenough, 2004, for reviews). Increases between $1 \%$ and $6 \%$ in total cortical weight were observed after enrichment in weanling aged rats (Rosenzweig et al., 1972). In the occipital cortex, enrichment-related changes were typically larger, in the 6-10\% range (Rosenzweig and Bennett, 1996). In adult rats, more specific interventions focusing on motor skill learning and exercise have been reported to result in a $10 \%$ increase of motor cortex thickness (Anderson et al., 2002; Diaz et al., 1994) and an almost 20\% increase of regional cerebellar volumes (Black et al., 1990). However, observed changes in volume and thickness do typically not exceed 10\% (Anderson, 2011). Experience-dependent changes in volume and thickness have also been observed in birds (Clayton and Krebs, 1994), with an effect size of $28 \%$, and mice (Lerch et al., 2011), with changes in striatal and hippocampal volume of about 3-4\%, in response to alterations in spatial navigation demands.

In 2004, Draganski and colleagues reported that experiencedependent morphological changes can also be observed in humans (Draganski et al., 2004). Voxel-based morphometry (VBM) was used in this study to analyze T1-weighted MR images collected before and after three months of juggling training. The training affected grey matter macrostructure in mid-temporal cortical areas and in left posterior intra-parietal sulcus. The study was seminal because it followed the same individuals over time in a longitudinal design. Previous studies had been cross-sectional, comparing the brains' of different individuals that have distinct past experiences (Amunts et al., 1997; Gaser and Schlaug, 2003; Maguire et al., 2000; Schlaug et al., 1995). Using a cross-sectional design it is impossible to determine whether observed structural differences are due to selection on the basis of genetic predispositions or to specific experiences. Pretest-posttest longitudinal studies are superior in this regard. Published studies of the latter kind that report analyses of T1weighted MR images, including their main methods and results, are listed in Inline Supplementary Table S1.

Inline Supplementary Table $\mathrm{S} 1$ can be found online at http://dx.doi.org/10.1016/j.neubiorev.2013.02.014.

An impressive number (33) of studies investigating experience-dependent macrostructural changes in human grey matter have accumulated in recent years. However, several of these studies suffer from serious flaws (see also Thomas and Baker, in press, for a review of some of these limitations). For example, many studies are using very liberal corrections of the alpha level (threshold for significance) for the multiple test problem in the mass univariate statistics approach that is a part of several techniques to automatically analyze grey matter structure (see e.g., Colom et al., 2012 and Kwok et al., 2011, using an uncorrected alpha level of .005 as threshold). In the absence of strong regional predictions or replication, such results are difficult to trust. Even more problematic than this, however, is the widespread practice of reporting effects without comparing results to those of a control group (of the studies in Inline Supplementary Table S1, the following used this procedure: Driemeyer et al., 2008; Granert et al., 2011; Gryga et al., 2012; Hamzei et al., 2012; Kim et al., 2010; Kwok et al., 2011; Landi et al., 2011; Langer et al., 2012; Stein et al., 2010; Teutsch et al., 2008; Thomas et al., 2009). These studies are difficult to interpret, because an unknown number of issues related to scanner stability over time and to confounding physiological effects such as hydration status (Duning et al., 2005; Kempton et al., 2009) can compromise measurement stability. In addition to controlling for these confounds, effects of physiological maturation and senescence must be controlled in interventions that last longer than a few days or weeks. Thus, it is only group by time interactions and net effects (changes in experimental group minus changes in control group) that can be safely interpreted. Note also that, for appropriate control of scanner drifts or other time-related effects, data for experimental and control groups must be collected close in time.

Surprisingly, some studies including control groups did not perform direct group by time analyses, but rather performed separate tests for time effects in the two groups (Woollett and Maguire, 2011), which is inappropriate (see Nieuwenhuis et al., 2011, for review), or did not clearly describe and report the results of interaction analyses (Draganski et al., 2004, 2006). At least one study (Ceccarelli et al., 2009) also seems to have collected data for experimental and control groups at different time points. In addition, several studies performed group by time analyses only as secondary analyses (applying more liberal significance thresholds) after regions-of-interests (ROIs) had been identified in whole-brain analyses for time effects in the experimental group (such studies include Bezzola et al., 2011; Hölzel et al., 2011; Ilg et al., 2008; Scholz et al., 2009; Taubert et al., 2010; Wenger et al., 2012). Such a non-independent selection procedure biases results toward finding a group by time effect and should be avoided (see Kriegeskorte et al., 2009, for a review of this issue). Nevertheless, a few of the studies that suffer from some of these methodological weaknesses have reported a predicted regional specificity of 
effects (e.g., Granert et al., 2011; Landi et al., 2011; Langer et al., 2012), which makes these results difficult to dismiss. However, other reports are more questionable, such as for example papers reporting experience-dependent changes in one direction (e.g., increases) in superior regions of the brain and changes of the other direction (e.g., decreases) in inferior regions (see e.g., Taubert et al., 2010, for an example). Such results could stem from time-related changes in the positioning of subjects in the magnet. That is, positioning changes within the barrel-shaped gradient distortions are likely to affect the superior and inferior parts of the brain in different directions (Caramanos et al., 2010; Jovicich et al., 2006). Clearly, future research must apply appropriate statistical analysis and use a control-group design, with groups measured close in time and handled identically during measurement.

Beyond design and statistical analyses, another weakness in this field is the heavy reliance on VBM to estimate structural changes in regional grey matter. VBM has several advantages and has been instrumental in research on experience-dependent structural brain change. However, it also has important weaknesses (for details on some of these limitations, see Bookstein, 2001; Davatzikos, 2004; Thomas and Baker, in press) and has proven highly sensitive to relatively small changes in the preprocessing pipeline (for a demonstration of this, see Senjem et al., 2005; Thomas et al., 2009). Most importantly, the key measure of VBM, the probability of each voxel in an image being grey matter, is not logically straightforward to relate to the macrostructure of the biological brain. For example, we do not know how the "probability of a voxel being grey matter" fit among traditional measures of the brain's morphology, such as cortical thickness, volume, and density of specific neural tissue within a given volume. Also when the images are modulated, that is, when the required normalization is encoded into the voxels, so that each voxel represents a mix of volume and grey matter probability (the Jacobian voxel compression map multiplied by grey matter probability), the interpretation in terms of the biological brain is unclear. Other techniques to assess changes on T1-weighted MR images, such as manual segmentation (Lövdén et al., 2012), automatic techniques to measure regional brain volume (with e.g., tools such as FreeSurfer or FSL-FIRST; Erickson et al., 2011; Mårtensson et al., 2012), and automatic outlining of cortical thickness (with e.g., tools such as CIVIC and FreeSurfer; Engvig et al., 2010; Haier et al., 2009; Langer et al., 2012; Mårtensson et al., 2012; Wenger et al., 2012) are superior in this regard. For example, cortical thickness measures map onto known thickness of the brain in a valid manner (Fischl and Dale, 2000). In contrast, VBM may produce a measure that reflects a mixture of morphological changes, including density, cortical surface area, cortical folding, and cortical thickness (e.g., Hutton et al., 2009). In addition to this uncertainty about the relationship between the measures of VBM and the brain, there is also widespread confusion in the literature regarding what VBM measures on the images per se. This becomes apparent when inspecting the labels that occupy the $Y$-axis in reports of experience-dependent grey matter changes. These include grey matter density (e.g., Driemeyer et al., 2008), grey matter concentration (e.g., Hölzel et al., 2011), grey matter intensity (e.g., Woollett and Maguire, 2011), grey matter signal intensity (e.g., Hamzei et al., 2012), grey matter volume (e.g., Takeuchi et al., 2011), and percentage signal change (with the denominator often unknown; e.g., Boyke et al., 2008). The units for these labels are typically not spelled out, but sometimes "percentage" (of what is often unclear; that is, the axis actually has no unit; e.g., Ilg et al., 2008) or "arbitrary units" (e.g., Driemeyer et al., 2008) are reported as units. Such uncertainty about labels and units of measurement is clearly unsatisfactory for a field. In sum, VBM clearly detects changes in images in a highly sensitive way and results may replicate across studies and samples (e.g., May and Gaser, 2012). VBM will therefore continue to be an important technique in this field, but because interpretations of observed effects are difficult, VBM must be complemented with other techniques.

In summary, methodological limitations plague some of the reported studies of experience-dependent structural brain plasticity. However, several studies are difficult to dismiss based on such concerns. For example, Erickson et al. (2011) report a solid study in which 120 older individuals were randomly assigned into an aerobic exercise group $(n=60)$ or to a stretching control group $(n=60)$. Participants completed the exercises three days a week. Hippocampal volume was automatically segmented on T1-weighted structural MR images collected before the intervention, six months into the intervention, and after a total of 12 months of intervention. The group by time interaction reached significance, such that aerobic exercise resulted in increased hippocampal volume of roughly $2 \%$ over the 12 -month period, whereas the control group displayed decrements of roughly $1.4 \%$ over the same period (see Fig. 1A and B). The observed decrements for the control group are in the range of normal age-related hippocampal shrinkage (Raz et al., 2005).

Reports of experience-dependent structural brain changes are not restricted to physical exercise and subcortical structures. Mårtensson et al. (2012) studied cortical thickness and hippocampal volumes of military conscript interpreters before and after three months of intense language studies. Relative to university student controls, interpreters showed selective increases in hippocampal volume (Fig. 1C), and in cortical thickness of the left middle frontal gyrus, inferior frontal gyrus, and superior temporal gyrus (Fig. 1D). Though the design is quasi-experimental and the threshold for significance in the whole brain analyses was liberal (.001, uncorrected), the findings that the peak cortical thickness increases for interpreters occurred in predicted regions known to be involved in language processes (for reviews, see Davis and Gaskell, 2009; Demonet et al., 2005; Price, 2010) makes it likely that the observed morphological changes are related to language acquisition. In addition, meaningful brain-behavior relations were observed within the group of interpreters, as several of these anatomical changes correlated with behavioral variables linked to language acquisition.

Overall, looking at the studies with acceptable quality that provide information on effect sizes, reported net effects of experience on hippocampal volume are in the $2-4 \%$ range (Erickson et al., 2011; Lövdén et al., 2012; Mårtensson et al., 2012). Cortical effects fall within the same range (2-5\%; Engvig et al., 2010; Mårtensson et al., 2012; Takeuchi et al., 2011). Though the magnitude of these effects sizes should be interpreted with caution, because only significant effects are considered, the upper limit of these effects indicate that experience-dependent effects on grey matter are likely to be relatively small.

\subsection{Microstructural changes underlying changes in regional grey matter volume and cortical thickness}

Soon after Rosenzweig and colleagues discovered the effects of enrichment on cortical thickness in rodents, questions arose regarding the microstructural changes that account for these effects. Research into the cellular effects of environmental enrichment and other more specific forms of learning therefore took off (Anderson, 2011; Markham and Greenough, 2004). It was discovered that environmental enrichment led to increases in dendritic length and branching in the visual cortex of rats (Green et al., 1983; Volkmar and Greenough, 1972). Changes in dendritic length and branching following enrichment extend across several brain regions, including hippocampus (Juraska et al., 1989), frontal cortex (Greenough et al., 1973), and temporal cortex (Greenough et al., 1973), and have also been observed in non-human primates (Kozorovitskiy et al., 2005). Because density of synapses can vary 

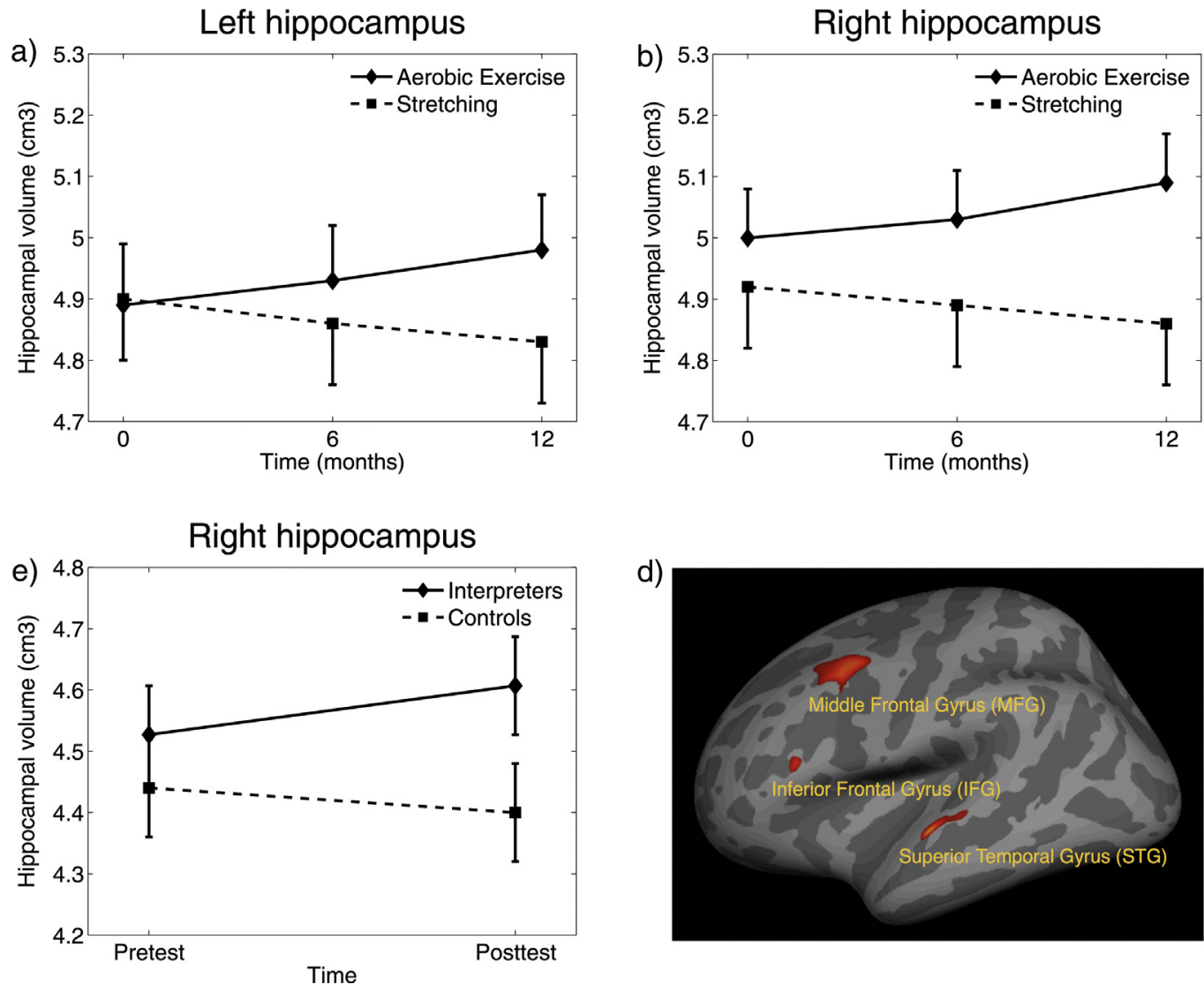

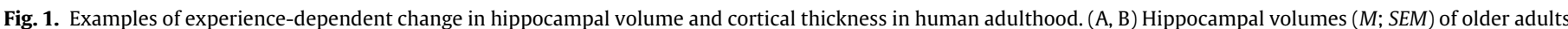

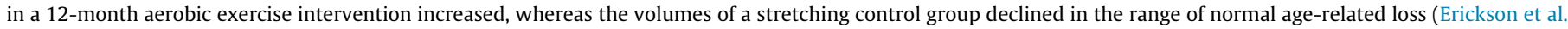

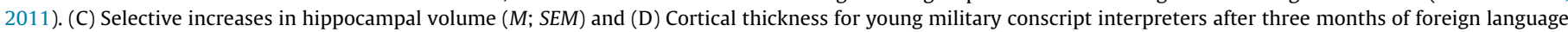
acquisition as compared to university student controls.

independently of dendritic length, changes in dendritic structure do not automatically translate into an increased number of synapses (Anderson, 2011; Kolb et al., 2008). However, a large body of literature has shown that environmental enrichment can also increase the number of synapses and dendritic spines per neuron in adult animals (Briones et al., 2004; Jones et al., 1997; Kozorovitskiy et al., 2005). Specific forms of training, such as motor and spatial learning, have been reported to result in similar effects on dendritic branching and synapse numbers (Black et al., 1990; Kleim et al., 1998; Kolb et al., 2008; Moser et al., 1994; Withers and Greenough, 1989). Note, however, that more recent studies using in vivo two-photon microscopy in mice, rather than static staining techniques such as the Golgi method, have shown that the large-scale organization of dendrites is relatively stable and that experience-dependent effects are restricted to alterations in synaptic connectivity as observed in growth and elimination of dendritic spines and axonal boutons ( $\mathrm{Fu}$ and Zuo, 2011; Holtmaat and Svoboda, 2009).

Experience-dependent synaptic plasticity, and other forms of microstructural brain plasticity, can obviously occur without changes in total regional volume, but when volume changes do occur, is it likely that synaptogenesis is the cause of volume changes? Few studies have addressed this issue, but a recent study by Lerch et al. (2011) provided a link between macrostructural changes, observed with high-resolution MR images, and microstructural changes. Mice training for five days in a cued version of the Morris water maze displayed volume growth in striatum, whereas mice training on a spatial variant of the maze displayed hippocampal growth. Immunohistochemistry revealed that GAP-43 staining, an indicator of changes in presynaptic morphology and probably also postsynaptic remodeling, correlated with volume changes. By contrast, markers of neuron and astrocyte numbers or sizes did not show such a link. Thus, synaptic remodeling remains a likely candidate of experience-dependent volume changes, but it seems highly unlikely that such small changes alone are sufficient to result in volume increases (Johansen-Berg et al., 2012). However, addition of synapses and changes in dendritic structure following enrichment and skill acquisition might be accompanied by both growth of capillaries and glia (Anderson et al., 1994; Black et al., 1990), perhaps to support increased energy demand of the new synapses. Total increase in tissue volume may thus reflect the combined increase of these related factors. A similar coupling has been observed between experience-dependent adult neurogenesis (Kempermann, 1997, 2008; Snyder and Cameron, 2012; van Praag et al., 2000), which seems to be restricted to the dentate gyrus of the hippocampus (Bhardwaj et al., 2006), and growth of capillaries (Palmer et al., 2000). Though learning may be primarily related to synaptogenesis and dendritic branching, physical exercise may affect capillaries without increasing neural tissue (Black et al., 1990). In addition, glia may increase in a compensatory manner to balance decreases in synapses (Tata et al., 2006). Thus, experience-dependent volumetric changes cannot always be assumed to originate from changes in the number of synapses per neuron. Note also that density (e.g., number of cells or synapses per unit volume) and total regional volume are two principally independent measures that must be considered together for understanding experience-dependent structural changes (Anderson, 2011). In principle, one could observe larger volume accompanied by decrease in a particular aspect of cell tissue density, stable cell tissue density (implying growth of, for example, dendrites), or increasing cell density (implying disproportional 
growth of cell tissue). Of course, unaltered or decreased volume may also be accompanied by decreased, stable, or increased density. Various types of tissue (e.g., dendrites, synaptic spines, and capillaries) can in principle trade off in these ways. Also, the fraction of extracellular to intracellular space (typically around 15-30\%; Sykova and Nicholson, 2008) may also change during, for example, development and aging, and in a short-term dynamic and activation-related manner due to swelling and shrinking of cells (e.g., astrocytes; Le Bihan, 2007; Sykova and Nicholson, 2008).

In sum, work on experience-dependent changes in animals suggests that learning-related volume alterations might be related to synaptogenesis and changes in dendritic morphology. In hippocampus, neurogenesis may also play a role. However, specific brain changes are unlikely to occur in isolation. Rather, plasticity of regional brain volume likely reflects a cascade of changes in dendritic branching, synapses, cell numbers, cell sizes, and capillaries. Also, one must consider both tissue density and volume as complementary measures when investigating changes in brain structure.

Since the early publications suggesting experience-dependent volume changes in humans (Amunts et al., 1997; Draganski et al., 2004, 2006; Maguire et al., 2000), speculations about the microstructural changes underlying these effects have filled the discussion sections of numerous papers. However, only a few empirical studies have attempted to address this issue empirically. Recognizing that volume and density provide complementary information, Lövdén et al. (2012) measured hippocampal volume on T1-weighted images and used diffusion-tensor imaging (DTI) to estimate barrier density in the grey matter of the hippocampus in younger and older men taking part in spatial navigation training. DTI measures diffusion of water molecules in tissue and has become an important technique for estimating white matter integrity, but the technique can also provide useful information about grey matter. When applied to grey matter, the primary measure of interest is mean diffusivity (i.e., the mean of diffusivity of molecules in the three estimated directions). This measure is sensitive to the hindrance of diffusion of molecules by various membranes (Beaulieu, 2002) and can therefore be considered as a measure of barrier density. It should however be noted that the technique can be limited by the relatively large voxel size needed (often around $2 \mathrm{~mm}$ isotropic), so that the risk for partial-volume effects has to be considered.

Lövdén et al. (2012) randomized participants into a training group that navigated in a virtual environment while walking on a normal exercise treadmill at a relatively modest and not physically demanding pace (Fig. 2A) or into a walk-time-yoked control group that walked on the treadmill without the virtual environment. A total of forty-two 50-min training sessions were administered over a period of four months. MR images were collected before (pretest), immediately after completion of training (posttest 1), and four months after the termination of training (posttest 2). Hippocampal volumes were manually traced on the T1-weighted MR images and mean diffusivity was determined for these volumes on the images obtained with DTI. Results showed a significant group by time interaction for hippocampal volume (Fig. 2B and C): Participants in navigation training displayed stable hippocampal volumes over time, whereas those in the walk-time-yoked control group showed volume decrements in the range of normal age-related decline. The effect sizes observed by Lövdén et al. (2012) resembled closely those reported by Erickson et al. (2011) for physical exercise (i.e., in the $2-4 \%$ range). Importantly, mean diffusivity in right hippocampus showed a selective decrease following spatial navigation training, indicating increased barrier density (see Fig. 2C). This decrease was not present in walkers, who rather tended to display increases in mean diffusivity, and returned to baseline four months after termination of training. In other words, navigators displayed a pattern of stable hippocampal volumes and increased barrier density (in right hippocampus), whereas walkers decreased in volume and barrier density. These findings suggest that experience-dependent effects on hippocampal volume do not stem from altered tissue fluid, but rather reflect effects on cell membranes that can act as diffusion barriers.

Using the same training paradigm, but focusing on younger men only, Lövdén et al. (2011) reported that navigation training resulted in increases in N-acetylaspartate (NAA), as measured with MRI, in the right hippocampus. These changes correlated with improvements in navigation performance. NAA is almost only available in neurons and therefore serves as a relatively specific marker of metabolic and functional aspects of neural tissue (Moffett et al., 2007). Training-related changes in NAA were absent in carriers of the Met substitution of the brain-derived-neurotrophic-factor (BDNF) gene, known to reduce activity-dependent secretion of BDNF, a neurotrophin involved in neural plasticity at several levels (Binder and Scharfman, 2004; Lu, 2003; Poo, 2001). Together with the results on hippocampal volume and barrier density (Lövdén et al., 2012), these results suggest that adult experience can affect neuronal microstructure in hippocampus, which in turn may contribute to observed effects on volume. Also, in line with much work on non-human animals, activity-dependent release of BDNF may play a mediating role in the effects of experience on brain structure in humans.

In summary, evidence on the microstructural changes behind experience-dependent changes in human brain volume is scarce. However, in line with animal work, human evidence suggests that volume changes may reflect neuronal alterations. Findings on micro-structural changes in humans are limited to the hippocampus, and it is unknown whether the results generalize to the neocortex. More evidence on this issue may come from integration of methods and paradigms in systems and molecular neuroscience (Zatorre et al., 2012), using the same paradigms and MRI measures in animals and humans, supplemented by histological measures in animals. Using complementary information from several neuroimaging techniques (Draganski et al., 2011) may also further our understanding of experience-dependent volume changes, and of brain plasticity in general.

\subsection{The time course of experience-dependent volume changes}

Studying the time-scale over which experience-dependent structural changes occur should help developing process models of how learning is implemented in the brain (May, 2011). Unfortunately, there is little systematic work on the time course of experience-dependent volume and thickness changes in non-human animals. In the classic enrichment paradigm, the manipulation typically lasts for at least a month and is constant (i.e., animals in enriched vs. non-enriched environments). However, enrichment for shorter time periods each day may also affect volume and thickness (Black et al., 1990; Rosenzweig et al., 1968). For example, in rats training motor skills, 30 days of 15 min learning per day increased regional volume in cerebellum (Black et al., 1990). However, much shorter periods of intervention may also result in volume changes. Lerch et al. (2011) found that five days of Morris water maze training (six trials per day) were sufficient to result in increases of regional grey matter volume in mice. Quallo et al. (2009) used VBM to analyze structural MR images collected from three adult macaque monkeys before ( 2 measurements), during ( 3 measurements), and after ( 1 measurement) learning to use a rake to retrieve food. Monkeys received intensive training each day over a period of 21 days. Learning-related increases were found in right superior temporal sulcus and secondary somatosensory area for two of the three monkeys. Interestingly, these changes in grey matter morphology mapped onto the learning curves; no grey 
a)

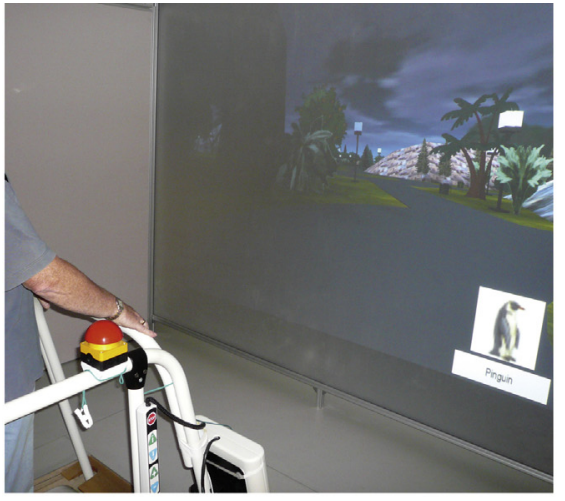

c)

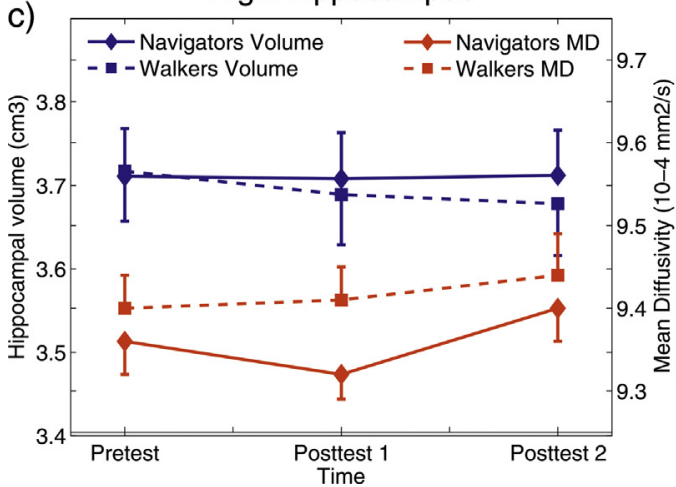

b)

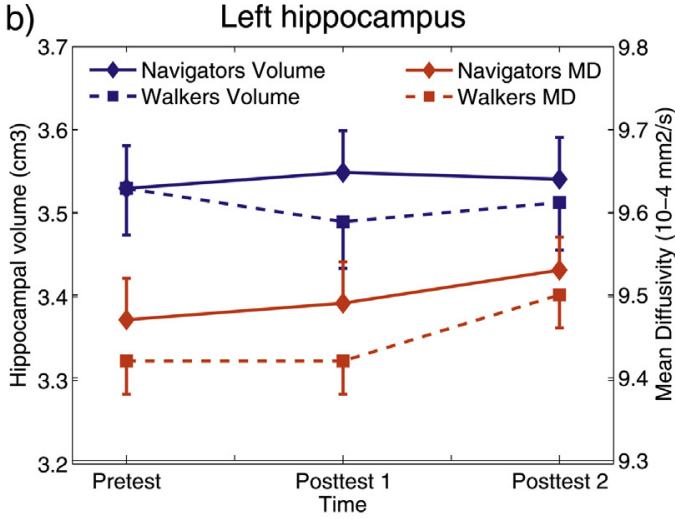

d)

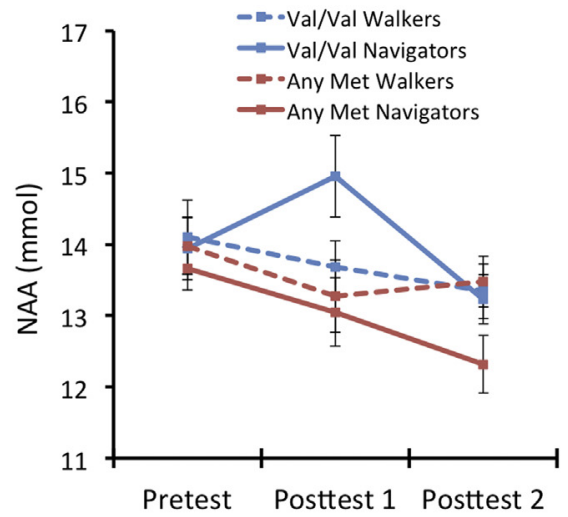

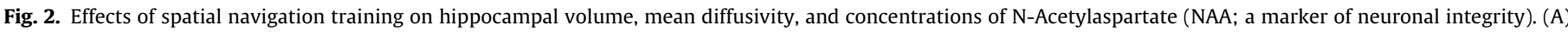

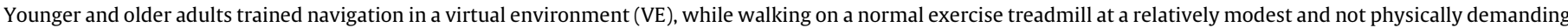

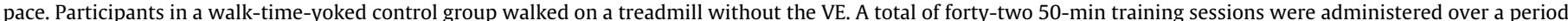

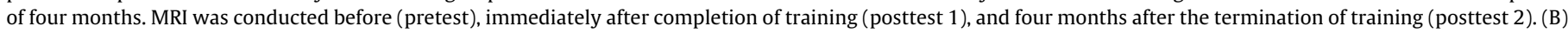

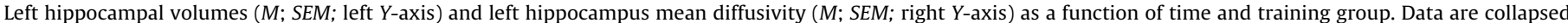

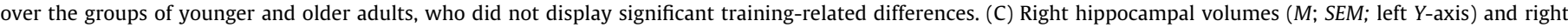

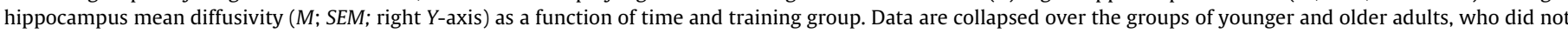

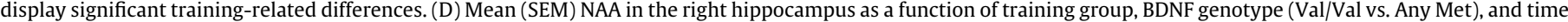
of assessment (pretest/posttest 1/posttest 2 ) in younger participants.

matter changes were observed in the monkey learning slowly and performing variably. Also, the increases in grey matter occurred earliest (within six days of training) in the monkey learning the fastest, and more slowly in a monkey with a flatter learning curve. Most interestingly, despite continued training, grey matter volume decreased after the monkeys' performance reached asymptote. Post training, the volume was bigger than before training, but much smaller in magnitude than the peak effect observed before asymptotic performance was reached.

The pattern of rapid expansion followed by partial renormalization, observed by Quallo et al. (2009), may signify a general principle of neural plasticity. Learning-related cortical map expansion also emerges relatively rapidly (e.g., within a few days), but then renormalizes after further behavioral training and despite stable performance (Molina-Luna et al., 2008; Reed et al., 2011). The initial expansion is thought to increase the pool of neural pathways from which the most efficient circuit can be selected. Perceptual and motor learning per se may occur in the selected circuitry, and the selection process results in a renormalized cortical map (Reed et al., 2011). The expansion-partial renormalization hypothesis is also supported by in vivo microscopic imaging studies of dendritic spines (see Fu and Zuo, 2011; Holtmaat and Svoboda, 2009, for review), indicating that a pool of new spines is generated early in learning - as early as after an hour of motor training (Xu et al., 2009; but see Kleim et al., 2004). This rapid increase of spines is followed by a slower (e.g., over several days) process of elimination of spines that existed before training, whereas new spines are selectively stabilized and remain several months after termination of training (Xu et al., 2009; see Fig. 3). The degree of spine formation is related to improvements in performance and survival of new spines is linked to maintenance of motor skill (Xu et al., 2009; Yang et al., 2009), indicating that new spines play a crucial role in learning. A similar process of expansion and selective stabilization is operating in learning-related neurogenesis (Dupret et al., 2007; Gould et al., 1999; Tronel et al., 2010). Note, however, that these various experience-dependent changes occur over very different periods, though the overall shape of their time course could be similar. For example, synaptogenesis and associated changes in dendritic spines can be formed over periods of minutes to hours, whereas neurogenesis may take days to weeks (Fu and Zuo, 2011; Holtmaat and Svoboda, 2009; Johansen-Berg et al., 2012; Zhao et al., 2008). That said, the contribution of neurogenesis to learning-related changes in volume of the dentate gyrus could be a faster process. Specifically, in contrasts to the primary effects of physical exercise on precursor cell proliferation, learning may affect the integration and survival of already more mature new neurons (Dupret et al., 2007; Fabel et al., 2009; Kempermann, 1997, 2008; Kempermann et al., 1998, 2002; Zhao et al., 2008). Addition of capillaries and astrocytes may, however, be processes that take days to weeks. In contrast, swelling of cells may be a very rapid process that occurs over seconds to minutes (Johansen-Berg et al., 2012; Le Bihan, 2007; Sykova and Nicholson, 2008). Finally, the pruning model of brain maturation is a prominent exemple of the pattern of expansion 

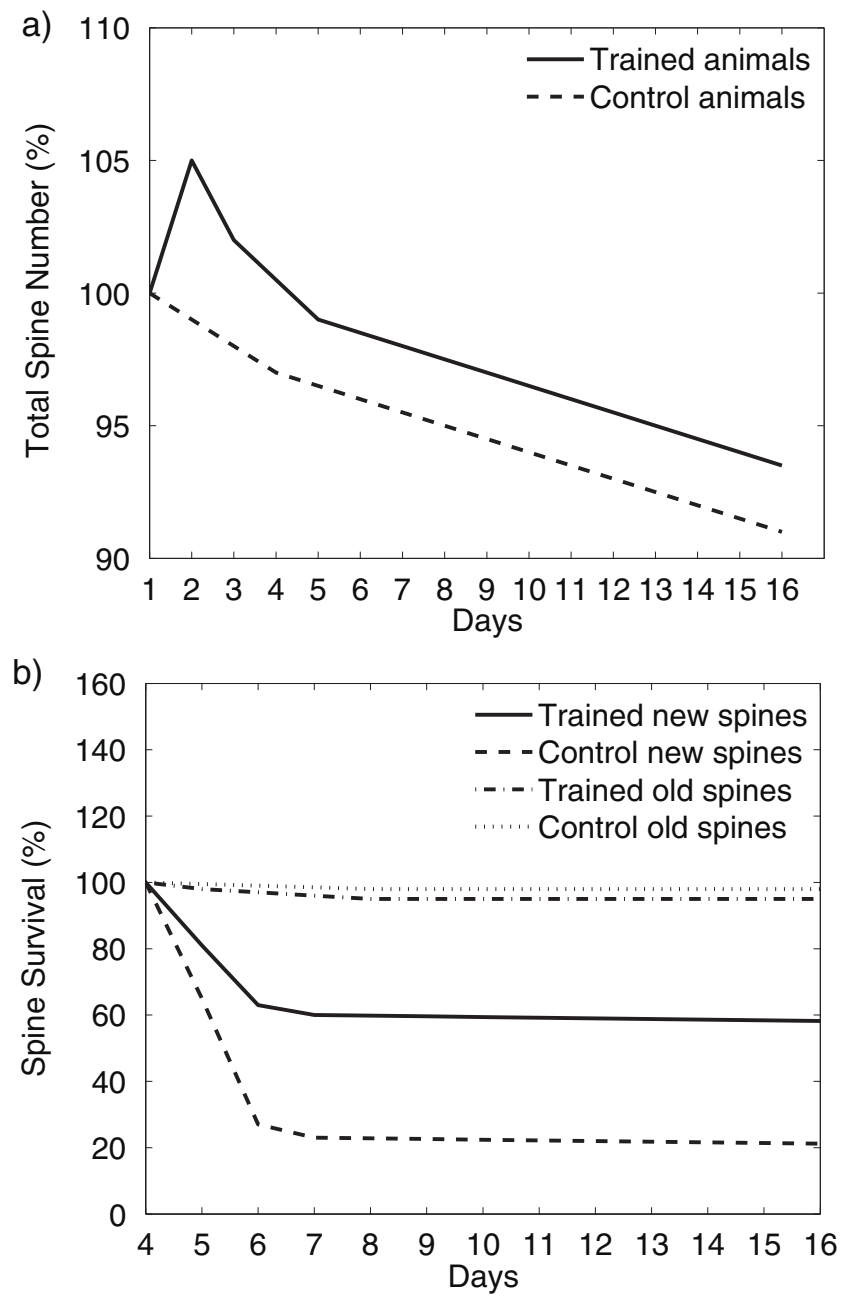

Fig. 3. Schematic portrayal of results from Xu et al. (2009) depicting number of dendritic spines in sensory and motor cortices (imaged in vivo) as a function of motor skill learning in adolescent mice (A) and spine survival over time and as a function whether the spines were formed during learning or preexisted (B).

followed by selective stabilization that operates over very long time. This model holds that mature neural networks are formed from an initial overproduction of a pool of connections that are then modified over years of development by experience, so that some are selectively stabilized and others are eliminated (Changeux and Dehaene, 1989; Cowan et al., 1984; Luo and O'Leary, 2005; Waimey and Cheng, 2006).

In sum, work on animals suggests that volume changes may occur relatively fast and after restricted daily training periods, that is, within a few days after the start of the intervention. Both work on volume changes as well as on experience-dependent microstructural changes support a general principle of expansion followed by selective stabilization and partial renormalization of overall density and volume to baseline levels, despite continued training. The initial overshoot is thought to be helpful for learning, but learning per se is reflected in the novel structure remaining after the selective stabilization process. We refer to this collection of predictions as the expansion-partial renormalization hypothesis (cf. Reed et al., 2011).

This expansion-partial renormalization hypothesis can be portrayed as a mechanistic specification of a more general supply-demand model of plasticity (Lövdén et al., 2010). In the latter model, which appears to be implicit in several studies of experience-dependent grey matter changes, brain structure is assumed to adapt to concurrent experiences. That is, manifestations of plasticity (e.g., volume changes) are considered as continuous, but sluggish, adaptions of the brain to meet the demands on supply (i.e., brain structure) coming from alterations in activity. This model is more abstract and general than the mechanistic and learning-specific expansion-partial renormalization model, which is mute with respect to predicting, for example, effects of disuse (e.g., limb immobilization). That is, the supply-demand model describes the driving force behind plastic changes both in situations when demand is higher than supply (e.g., learning situations) and when demand is lower than supply (e.g., disuse situations). The model clearly predicts decreases of regional volume in task-related areas in disuse situations (Lövdén et al., 2010) and such decreases have been observed in several studies (Granert et al., 2011; Langer et al., 2012).

Relatively little is known about the time course of experience-dependent volume and thickness changes in humans. Across studies there are large variations in intensiveness and duration of the interventions (see Inline Supplementary Table S1). Cortical changes have been observed after extensive interventions, such as three months of juggling (Draganski et al., 2004), three months of intense language studies (Mårtensson et al., 2012), and six months of aerobic exercise (Colcombe et al., 2006). Effects on hippocampal volumes have been observed after three months of foreign language learning (Mårtensson et al., 2012), four months of training spatial navigation every other day (Lövdén et al., 2012), and 12 months of aerobic exercise training (Erickson et al., 2011). However, changes might emerge much faster than this. For example, using VBM, Kwok et al. (2011) reported morphological changes in visual cortex after as little as $2 \mathrm{~h}$ (spread out over three days) of learning named subcategories of universal color names. Changes in ventral striatum have been observed after 3 days of practicing ( 30 min each day) writing the signature with the non-dominant left hand (Hamzei et al., 2012). Also, Teutsch et al. (2008) reported increases in middle cingulate cortex after one week of daily pain stimulation sessions and Driemeyer et al. (2008) found that one week of juggling training was enough to induce grey matter changes in occipital-temporal cortex. Although these studies are consistent with animal studies documenting rapidly emerging changes in grey matter, the four studies reporting such changes all lacked a control group. Of the studies directly comparing experimental and control groups in a valid manner, Takeuchi et al. (2011) reported the fastest appearing changes in grey matter. Using VBM, the authors observed that 5 days of 4-h sessions of mental multiplication and addition training resulted in trends for decreases in grey matter in fronto-parietal areas as well as the superior temporal gyrus for the experimental group, as compared with trends for increases in a placebo control group and a no-contact control group. To aid interpretation of these results, it would have been interesting to see measures of barrier density (e.g., mean diffusivity) and cortical thickness in these areas. Of the studies reporting increases, and using appropriate design and statistical analyses, Engvig et al. (2010) demonstrated the fastest emerging changes. These authors showed that eight weeks of learning a mnemonic technique (with a 1-h class per week and 4 days of home exercises) resulted in thickening of the right insula among older adults.

Hence, evidence indicates that cortical and subcortical experience-dependent changes in humans can be observed after a few months of intervention. Several studies also suggest that such changes may emerge much faster than this (i.e., within days or weeks), but the results of these studies need replication. Other findings do indeed suggest that regional volume may change dynamically over very short time scales. For example, drug-induced (haloperidol) acute dopamine (D2) receptor blockade resulted in transient volume changes in human striatum within hours (Tost et al., 2010). In another paper, Sagi et al. (2012) reported 
that short-term $(2 \mathrm{~h})$ spatial learning decreased mean diffusivity of the human hippocampus, indicating increased barrier density, although no volume changes were observed. Interestingly, a parallel experiment conducted with rats also revealed decreases in hippocampal mean diffusivity following $2 \mathrm{~h}$ of spatial learning. In addition, histology showed increases in BDNF levels and the amount of synaptic vesicles, and demonstrated alterations in the activation of astrocytes, suggesting that the rapid changes may reflect cellular swelling or changes in the ratio between intracellular and extracellular volumes (Le Bihan, 2007; Sykova and Nicholson, 2008). Again, these findings emphasize that density and volume are in principle two independent measures (Anderson, 2011).

The extremely rapid, almost immediate, nature of these changes suggests that these effects are of a different nature than the effects on volume and thickness observed over months in non-human and human learning. However, it is possible that short-term effects confound estimates of long-term effects. For example, a session just before the post-training examination in the scanner may distort pretest-posttest comparisons, because no training session was conducted just before pretest. In such situations, long-term and short-term activation-dependent effects are difficult to disentangle. Clearly, more work with greater methodological sophistication needs to investigate the time-scale of experience-dependent plasticity. For example, MR measurements could be conducted before and after training at both pretest and posttest, which would allow for disentangling short and long-term effects of experience. Also, it may be necessary to attempt to standardize functional activity (e.g., keeping eyes open vs. eyes closed constant, or assign a low-level cognitive task to subjects) during measurement of structural images. Currently, the effects of activity on the contrast of T1-weighted images are unknown, and such effects may also differ across different MR sequences.

A few studies have conducted measurements during, and not only before and after, periods of interventions and training. In the study by Erickson et al. (2011), effects of physical exercise on hippocampal volume were evident after six months, and increased linearly over time (baseline, 6 months, 12 months; see Fig. 1). Studies using multiple MRI measurements during learning have also been reported (Driemeyer et al., 2008; Hamzei et al., 2012; Taubert et al., 2010; Teutsch et al., 2008). However, in these investigations, it is difficult to interpret the exact time course of changes in morphology because of non-independent selection (Kriegeskorte et al., 2009) of the Region-Of-Interest (ROI) that provide the data on the time-course. That is, a ROI has been selected from whole-brain data based on a specific time contrast (e.g., between pretest and one of the other measurements), and data from the rest of the measurements are extracted from this ROI. In such a procedure, the ROI that displays data that best fit the originally specified statistical hypothesis will be selected. The study of Driemeyer et al. (2008) nicely demonstrates how this may bias the data toward a particular time course. In one analysis, data was extracted from a ROI formed after an analysis comparing baseline with the mean of three measurements conducted during juggling training. In another analysis, data was extracted from a ROI based on a statistical comparison between baseline and the first measurement during training. The time courses in question look very different: Data from the first ROI show rapid increases and then stability during training (in line with the hypothesis used to select the ROI). Data from the second ROI display a rapid increase to the first measurement followed by partial return to baseline during the rest of the training period (again in line with the hypothesis selecting the ROI). Clearly, future studies on the time course of experience-dependent changes in brain volume must select ROIs in a methodologically sound manner. For example, ROIs could be selected based on a priori considerations about the functional architecture of the brain, or based on functional activation patterns at one point in time.

In addition to effects of learning, maintenance of experience-dependent changes in human brain morphology after termination of training has been investigated in several studies. The evidence is mixed, with studies reporting decreases (Boyke et al., 2008; Draganski et al., 2004; Driemeyer et al., 2008), continuation of increases (Draganski et al., 2006; Scholz et al., 2009), or maintainance (Lövdén et al., 2012) of structural grey matter changes after termination of various interventions such as medical studies (Draganski et al., 2006), navigation training (Lövdén et al., 2012), and juggling (Boyke et al., 2008; Draganski et al., 2004; Driemeyer et al., 2008; Scholz et al., 2009). Also, methodological issues (e.g., non-independent ROI selection, reporting of select contrasts, such as quadratic effects only, and lack of appropriate control group comparisons) cloud interpretation of several of these reports.

In summary, the time course of experience-dependent brain-volume changes in humans remains unknown. Theoretical models and animal work (Fu and Zuo, 2011; Holtmaat and Svoboda, 2009; Molina-Luna et al., 2008; Quallo et al., 2009; Reed et al., 2011) predict that regional volumes may rapidly expand during learning and then partially renormalize, despite continued training. Future studies should examine these predictions. Such investigations may advance our understanding of the mechanisms regulating experience-dependent changes in regional brain volume (May, 2011). In such investigations, it is also important to take functional changes into consideration - an issue largely neglected so far. Specifically, in the course of learning, brain activation may decrease in some areas and increase in others (Dayan and Cohen, 2011; Kelly and Garavan, 2005), reflecting changes in how the cognitive task is processed, and such activation changes may influence the time course of learning-related changes in structural brain volume. In addition, it is important to use a variety of imaging modalities because different structural correlates of changes in experience are likely to follow different time courses.

\subsection{Are experience-dependent volume changes related to changes in behavior?}

It is tempting to think that volume and thickness changes are in some way reflecting learning. However, they could also be adaptations of the brain to changes in metabolic demands driven by novel levels of neural activity. That is, though potentially, but not necessarily, related to improvements in performance, they may not constitute the neural basis of learning per se (i.e., consolidated memory traces of past experiences). Changes of both types could be viewed as plasticity (Black et al., 1990; Lövdén et al., 2010), but in one case structural changes are assumed to be correlates of knowledge or skill, whereas in the other case they merely reflect adaptations (e.g., growth of capillaries) to changes in activity. Early animal work using the environmental enrichment paradigm addressed this issue. These studies first ruled out that changes in volume, thickness, or other structural alterations were due to confounding factors, such as changes in body size, stress, or social interaction (Grossman et al., 2002; Markham and Greenough, 2004). Reports of activity-specific regional brain changes have been convincing in this regard. For example, micro-morphological changes were found to be restricted to the "trained" hemisphere in sensory and sensorimotor paradigms, in which hemisphere-specific effects could be predicted (Grossman et al., 2002; Markham and Greenough, 2004). More recently, Lerch et al. (2011) demonstrated that mice training for five days on a cued version of the Morris water maze displayed volume growth in striatum, 
known from functional studies to support cue-based learning. Mice training on a spatial variant of the maze displayed growth of hippocampus, known to support learning based on allocentric spatial processes. Thus, structural changes are activity-dependent, but this does not preempt the answer to the question of whether learning, or merely activity, is needed for these changes to occur. Black et al. (1990) addressed this issue by comparing rats trained on a motor skill-learning task with rats exercising on a treadmill. Cerebellar volume changes and increases in synapses per neuron were observed only after skill learning, whereas increases in the density of blood vessels were observed only after exercise (see also Kleim et al., 1996). In contrast, Andersson and colleagues (Anderson et al., 2002) reported that both motor skill learning and exercise caused thickness changes in motor cortex of rats. The evidence on associations between changes in volume and performance is also mixed. Correlations between structural changes and learning outcomes have been observed in recent imaging work for dendritic spines (Fu and Zuo, 2011; Xu et al., 2009; Yang et al., 2009). In contrast, Lerch et al. (2011) found that changes in striatal and hippocampal volume were unrelated to performance gains in the Morris water maze task.

Thus, it appears that changes in synaptic morphology may be related to learning, but it is unknown whether volume and thickness changes are induced by alterations in regional levels of neural activity, or whether they specifically require acquisition of skill and knowledge. However, animal work gives some hints as to which questions to ask to the data on humans. For example, if learning is a crucial component, then interventions that entail a more pronounced learning component should result in larger structural changes than interventions that result in less learning. Also, individual differences in learning success should relate to structural changes even when individual differences in the time spent on task (as a proxy of the amount of activation in the brain) have been kept constant experimentally, or controlled statistically, across individuals. In contrast, if neural activity as such, independent of learning, is the crucial factor causing volume and thickness changes, then no differences in the effects of interventions varying in learning demands should be observed. Moreover, training time should account for individual differences in the amount of structural change.

Several human studies confirm that volume and thickness changes can be specific to those brain regions that are functionally relevant for the task at hand. Ilg et al. (2008) studied the effects of mirror reading training and also localized activity related to mirror reading with functional MR imaging. There were changes in grey matter morphology, as observed with VBM, in close proximity to the peak reading-related activation. Mårtensson et al. (2012) observed primarily left-lateralized changes in cortical thickness of the inferior frontal gyrus, mid-posterior superior temporal gurus, and dorsal middle frontal gurus after intensive acquisition of a foreign language (see Fig. 1D). These brain regions have been linked to language processing in numerous studies (Demonet et al., 2005; Price, 2010). Other demonstrations of regional specificity come from studies of motor skill, in which hemisphere-specific effects were predicted and observed (Granert et al., 2011; Landi et al., 2011; Langer et al., 2012). Granert and colleagues reported that immobilization of the right hand of patients with writer's cramp resulted in grey matter probability decreases in the contralateral left primary motor cortex (near the typical area representing the hand). Subsequent alleviation of constraint and addition of sensorimotor training caused returns to the original grey matter probability. Grey matter probability in right primary motor cortex tended to show the opposite pattern of effects, with increases followed by decreases. Though control groups and direct statistical comparisons across brain regions were absent, the results of this study suggest that volume and thickness changes may be activity-specific.

There are no systematic studies that attempt to disentangle the contribution of activity and learning success to changes in cortical thickness and volume. However, a few studies have examined the association between time spent on training and structural changes. Bezzola et al. (2011) reported that between-person differences in training intensity (i.e., the time period needed to complete 40 training session) was related to increases in grey matter, as observed with VBM, in the parieto-occipital junction of adults learning to play golf. However, studies directly investigating the association between amount of training and changes in grey matter structure have failed to observe significant effects (Boyke et al., 2008; Driemeyer et al., 2008; Hölzel et al., 2011). The association between training-related benefits and changes in grey matter morphology is also weak or non-existent (Boyke et al., 2008; Draganski et al., 2006; Driemeyer et al., 2008; Hölzel et al., 2011; Lövdén et al. 2012; Schmidt-Wilcke et al., 2010; Wenger et al., 2012). Issues such as statistical power, limited between-person differences in change, and low reliability of change scores come to front when interpreting these null findings, but it is clear that the bulk of studies fail to link learning to changes in human macro-scale brain morphology. A few exceptions exist, however. For example, Engvig et al. (2010) reported an association $(r=.37)$ between memory improvements and thickening of right fusiform cortex following training in a mnemonic technique. Landi et al. (2011) found that training on a visuomotor task resulted in increments in grey matter of the primary motor cortex that were related $(r=.77)$ to faster visuomotor adaption (i.e., higher performance) one year later. Finally, in the study of military interpreters, Mårtensson et al. (2012) demonstrated a relationship of acquired language proficiency to changes in hippocampal volume and thickness of superior temporal gyrus, indicating that individuals with larger structural increases learned faster (see Fig. 4A-C). Interestingly, interpreters who were rated by their teachers to struggle relatively more to acquire the learning goals tended to show smaller increases in these regions (Fig. 4A). This pattern of findings suggests that plastic changes in these regions are related to learning success, independently of training effort. However, it cannot be excluded that individuals learning less and showing smaller structural changes activated the hippocampus and the superior temporal gyrus less during language acquisition. On this view, individual differences in functional activation may partly account for individual differences in the talent for acquiring a language. The suggestion that individual differences in functional aspects matter is supported by the finding that interpreters struggling more to master the language displayed larger grey matter increases in dorsal middle frontal gyrus. This region overlaps with premotor language areas implicated in planning and top-down control of articulatory processes (Callan et al., 2004; Hickok and Poeppel, 2007; Meister et al., 2007). For those struggling relatively more to master a new language, the demands on such top-down support may have been elevated, leading to increased cortical thickness.

We conclude that macro-scale morphological changes are probably regionally specific in a way that relates to the cognitive processes that have been activated during training (Lerch et al., 2011; Mårtensson et al., 2012). However, based on available data, it cannot be clearly established whether learning is a necessary component for volume and thickness changes to occur, or whether changes in the amount of activity in a brain region are sufficient. Future studies on this issue need to collect functional imaging data to concurrently investigate individual differences in functional activity together with structural changes. Because individual differences in learning success, time on training, and functional activations are likely to be highly related in several situations, it is necessary to specify statistical models representing competing 
a)

a) 0.8

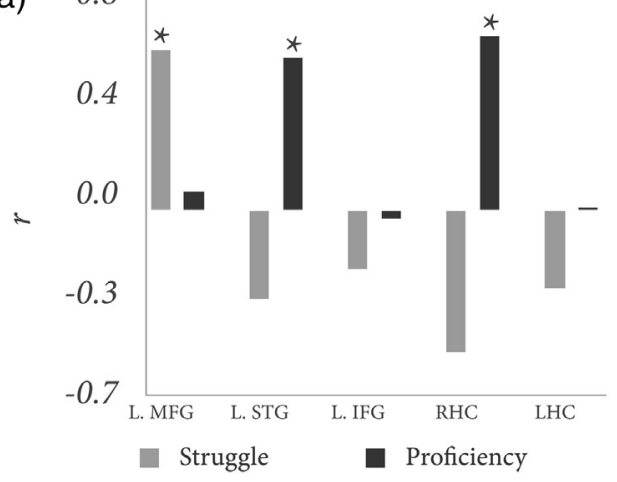

c)

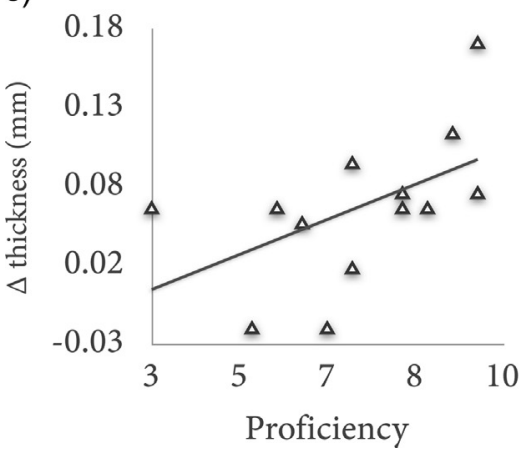

b)

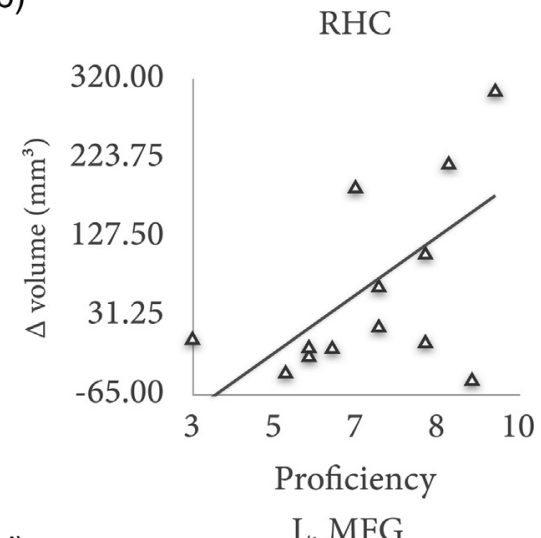

d)

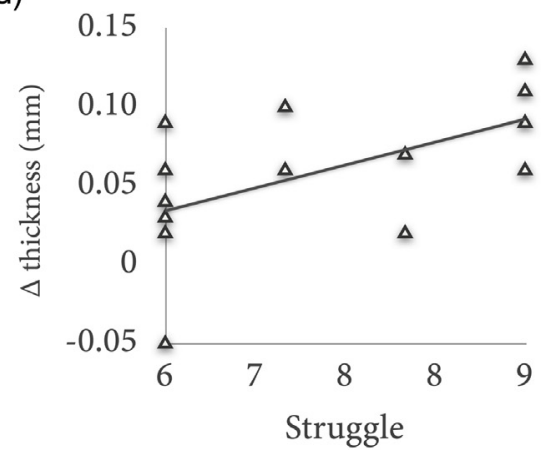

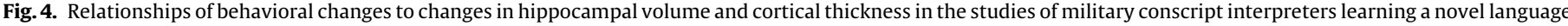

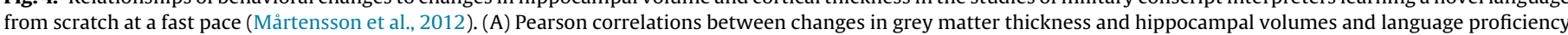

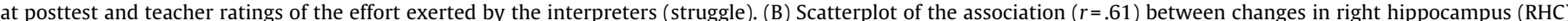

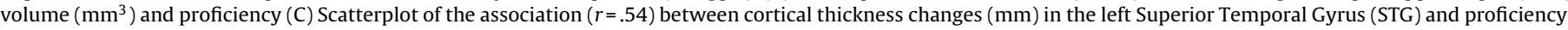
(D) Scatterplot of the association $(r=.56)$ between cortical thickness changes $(\mathrm{mm})$ in the Middle Frontal Gyrus (MFG) and struggle.

hypotheses and, if possible, to experimentally de-confound the variables of interest. Also, considering that learning may relate in complex and non-linear ways to micro-scale morphological changes (see Section 2.3), non-linear relationships between volume changes and behavioral outcomes should be considered. These and the other considerations described above imply that future studies require larger sample sizes than virtually all of the relevant studies performed thus far. Finally, different types of microstructural changes may relate differentially to learning and activity (Black et al., 1990; Kleim et al., 1996). Thus, there are reasons to reiterate the need for using complementary information from several imaging techniques (Draganski et al., 2011; Lövdén et al., 2012) and to integrate methods across the systems and molecular neurosciences (Zatorre et al., 2012) to better understand the nature of experience-dependent volume and thickness changes.

\section{Structural brain plasticity in adult development}

What role does plasticity of the brain play in adult development and aging? Epidemiological work indicates that a lifestyle rich in mental and physical challenges is associated with maintained cognitive functioning in old age (Fratiglioni et al., 2004; Hertzog et al., 2009; Lövdén et al., 2005). For example, higher complexity of main lifetime occupation is associated with higher cognitive performance in old age, but these effects are reduced after retirement, supporting the "use-it-or-lose-it" adage (Finkel et al., 2009). It is tempting to suggest that such findings stem from direct effects of experience that maintain brain integrity in aging (Lövdén et al., 2010; Nyberg et al., 2012). Yet, indirect effects may also play a role. For example, an engaged lifestyle may reduce the likelihood for negative effects (e.g., depression, stress, vascular conditions) on brain and cognition (Lövdén et al., 2010). Directly investigating the effects of experience on brain structure in experimental paradigms, and thus better understand the mechanisms behind the effects of lifestyle on adult development and aging, is one way to approach these confounds. For example, if plasticity plays a role for individual differences in aging, then the brains of older adults should continue to show structural plasticity in response to learning and experience. However, given decreases of grey matter volume and cortical thickness in aging (Fjell et al., 2009; Raz et al., 2005), older adults may display reduced experience-dependent brain changes. Also, when studying aging, maintaining brain volume following an experimental intervention along with declining volume in control groups may also qualify as instances of plasticity (e.g., Lövdén et al., 2012). That is, intervention-induced plastic changes may compensate for the degenerative changes causing reduction in brain volume. However, we note that such an empirical pattern may also arise from intervention-induced increases of maintenance factors (e.g., repair processes) that prevent loss in the first place, which may be viewed as qualitatively different effect from that produced by plasticity. Nevertheless, intervention-induced effects on structure in old age must be studied against the background of age-related changes in brain volume and when comparing the magnitude of effects in different age groups, the net effect (change in experimental group - controls) is the key dependent variable. With this consideration in mind, we review the evidence on adult age differences in macro-scale structural brain plasticity. 


\subsection{Adult age differences in experience-dependent volume and thickness changes}

Animal models suggest that increasing age is associated with reductions in the capacity for adaptive changes. For example, Wagner et al. (2000) found that the aged rat brain responded more slowly and to a lesser extent to chemically induced seizures compared to the young rat brain. A decreased coordination of genes involved in brain plasticity with increasing age may be one contributing factor to the reduced potential for experience-induced change (Kirkwood, 2005). As noted, dendritic spines could play an important role in structural plasticity in the adult brain (Fu and Zuo, 2011; Holtmaat and Svoboda, 2009; Kasai et al., 2003). Dumitriu et al. (2010) found that changes, especially in thin spine morphology, are related to the ability to perform a delayed non-matching-to-sample task. They concluded that age-related impairments in learning partly reflect changes in thin spine plasticity. Bloss et al. (2011) reported an interesting experiment using the negative impact of stress on dendritic spines to demonstrate plastic changes in different age groups. In young rats, stress resulted in dendritic spine loss and altered patterns of spine morphology. In contrast, spines from middle-aged and aged animals were remarkably stable and did not show evidence of remodeling as a function of stress. These data provide evidence that experience-dependent spine plasticity is altered by aging and, together with other work, support a model in which dendritic spines become progressively less plastic and more stable in aging (Bloss et al., 2011; Grutzendler et al., 2002; Holtmaat et al., 2005; Yang et al., 2009).

Nevertheless, it has been repeatedly shown that reorganization in various areas in adult brains can occur. Neural systems are modifiable networks throughout the lifespan, and this property is not limited to the early phases of development or young adulthood but can also extend into late life. In the following, we will summarize evidence from both animal and human research, demonstrating changes in brain structure in old age.

The animal literature shows cognitive benefits and neural reorganization from exercise in older rodents, for example effects of long-term voluntary exercise in running wheels (Nithianantharajah and Hannan, 2009; van Praag et al., 2000) and treadmill training (Aguiar et al., 2011; Gomez-Pinilla et al., 1998). In addition to exercise, environmental enrichment has induced changes in dendritic structure among middle-aged rats (Green et al., 1983) and in spiny branchlets of cerebellar Purkinje neurons in aged rats (Greenough et al., 1986). Enriched housing can even return the cells of old animals to the level of young animals living in impoverished cages (Kolb et al., 1998). An interesting question concerns whether a longer period of housing could stimulate a degree of growth similar to that seen in the younger enriched animals, or whether this growth is always less pronounced in aged compared to young animals. Also the age at which animals are placed in enriched environments has qualitatively different effects on dendritic structure. Remarkably, Kolb et al. (1998) found similar results in both younger and senescent animals, namely increases in dendritic length and spine density following enriched housing. Juvenile animals, however, exhibited an increase in dendritic branching, but a consistent decrease in spine density. The animals thus showed a change in distribution of synapses on pyramidal neurons that differed markedly from that of older animals. These examples underscore that enriched experience can have multifold effects on brain structure, and may result in different functional consequences at different ages. Kempermann et al. (1998, 2002) extended research on age differences in plasticity from changes in dendritic spines to neurogenesis. This research demonstrates that, although neurogenesis in the dentate gyrus generally decreases with increasing age, stimulating adult and aged mice by switching to an enriched environment results in increased survival of new neurons, accompanied by improvement in behavioral performance.

A handful of studies report plastic changes in the aged human brain. Colcombe et al. (2006) randomly assigned older adults (60-79 years) to a cardiovascular training program or a stretching and toning program for six months. Older adults in the cardiovascular training group exhibited increases in anterior white matter volume and for gray-matter volume (assessed with VBM) in inferior frontal gyrus, anterior cingulate, and superior temporal gyrus. In another study, elderly persons who were trained to learn three-ball cascade juggling showed grey matter changes (using VBM) in brain regions related to skill acquisition in the middle temporal area of visual cortex, in left hippocampus and in nucleus accumbens bilaterally (Boyke et al., 2008). Behavioral gains in the old subjects were smaller than in a 20-year old group in a previous study trained on the same paradigm (Draganski et al., 2004). The authors describe the training-related middle temporal changes in their older adults as "slightly smaller" than those seen in the previous study with younger adults, but do not state whether those age differences were significant. In addition, the older adults showed training-related changes in hippocampus and nucleus accumbens, two regions that had not shown alterations in young adults. However, as the two age groups were investigated in two different studies, the reported brain changes cannot be directly compared. Engvig et al. (2010) investigated 22 middle-aged and older healthy adults between 42 and 76 years who underwent an 8-week training regimen in the Method of Loci aiming at improving verbal source memory. Compared to controls, trainees showed regional increase in cortical thickness in right insula, left lateral orbitofrontal cortex, and fusiform cortex. Thickness change in right fusiform and lateral orbitofrontal cortex correlated positively with the degree of improvement in memory performance. Unfortunately, the authors do not report whether the age of participants correlated with thickness change. Considering the wide age range present in their sample, this could have elucidated the potential influence of chronological age on plastic changes.

Also in humans, aerobic exercise training has been shown to increase the size of the hippocampus in participants with a mean age of 67 years, whereas hippocampal volume declined in a stretching control group (see Fig. 1; Erickson et al., 2011). Further, after $40 \mathrm{~h}$ of golf practice, performed as a leisure activity, gray matter increases as measured with VBM were found in task-relevant cortical regions including sensorimotor regions and areas in the dorsal stream in a group of 40-60-year olds (Bezzola et al., 2011).

We have reported that prolonged spatial navigation training can protect hippocampal integrity against age-related decline in both younger and older adults who navigated in a virtual environment while walking on a treadmill for 50 min every other day over a period of four months (Lövdén et al., 2012). Interestingly, training-related cortical thickening in precuneus and paracentral lobule were observed in young (20-30 years), but not in old (60-70 years), navigators (Wenger et al., 2012). Thus, spatial navigation training appears to affect the hippocampus irrespective of age, whereas the potential for experience-dependent cortical alterations seems to decline with advancing adult age. Again, this underscores the necessity to differentiate between neuroplasticity in different brain regions, as different mechanisms might be at work depending on area.

In sum, evidence from animal and human research suggests that changes in structural plasticity during adulthood and old age are gradual, rather than abrupt. It is impossible to unequivocally state whether experience-dependent brain changes are reduced in late relative to early adulthood. Age-comparative studies that investigate both younger and older adults in the same training paradigm in the same study are scarce. Instead, most studies show structural 
plasticity either in young or old adults. Thus, the extent to which the aged brain harbors the potential to exhibit plastic changes relative to a young adult brain is difficult to gauge. More studies incorporating a wide age range of participants or at least two or three adult age groups plus appropriate control groups are warranted to further explore the premises that need to be fulfilled in different brain regions (e.g., hippocampus vs. neocortical regions) within aging brains to provide the continued potential for plastic change.

\section{Plasticity in the adult brain: Research recommendations and open issues}

Measurements of adult brain structure have revealed several examples of experience-dependent plasticity. Studying experience-dependent changes with MR images provides an attractive window into understanding learning and adult development in humans. Though this field is plagued with several methodological limitations, there is now ample support from both animal and human studies that experience-dependent volume and cortical thickness changes can be detected with MR imaging. Now, the time has come to focus on studying the mechanisms, including the function, sequential progression, and microstructural nature, of experience-dependent changes in regional brain volumes. In conducting this research, the following considerations are worth considering.

First, to delineate the progression of structural plasticity and explore its functional significance for changes in behavior, we need studies with relatively large samples (e.g., at least 30 individuals per group). These studies should feature a multi-modal imaging protocol to capture the dynamic and multidimensional nature of plastic changes in various aspects of brain tissue, including neurons, glia, and capillaries. In particular, we recommend the joint use structural MRI, diffusion imaging, MR Spectroscopy (MRS), and measures of blood flow and volume (e.g., arterial spin labeling) because these modalities provide complementary evidence that lead to a clearer picture of the mechanisms involved. For example, in the Lövdén et al. (2012) study, documenting simultaneous effects on volume and diffusivity strengthened the claim that the maintenance of hippocampal volumes in the training group may be associated with effects on cell membranes. Lövdén et al. (2011) used MRS to further augment this point, suggesting that the cells targeted by DTI may, in fact, include neurons. It is still a technical challenge to combine different imaging modalities (e.g., due to different voxel sizes and co-registration issues), but solving these challenges may be worthwhile considering the benefits in explanatory power.

Second, the broadening of imaging modalities needs to be accompanied by an increase in temporal resolution. The Nyquist theorem states that periodic processes need to be assessed at least at twice their frequency to be detectable under ideal conditions. Given that the rate and shape of most of the mechanisms contributing to plasticity are not sufficiently understood, strong statements about the appropriate spacing of measurement occasions are difficult to make at this point. However, it seems safe to assume that the pretest-posttest design typically used in human intervention research provides an impoverished picture of plastic changes in the human brain, such that many transient aspects of structural change may simply not be noticed in the first place. Studies with frequent assessments of structural brain properties before, during, and after the intervention are needed to provide a sound empirical basis for exploring mechanisms and testing theories of plasticity. Such work would also generate data in behaving humans that relate to the expansion-partial renormalization hypothesis. According to this hypothesis, an essential feature of structural plasticity is a temporary increase in the pool of neural pathways from which the most efficient circuits are selected in a later step. Processes related to the expansion part of this process may not be observable unless imaging data are obtained during early skill acquisition and compared to those from later phases.

Third, little is known about how mechanisms promoting structural maintenance in adulthood and old age relate to mechanisms promoting plasticity in the sense of growth (Lindenberger et al., 2012; Nyberg et al., 2012). Helping an organism to prevent decline may primarily consist in strengthening mechanisms that conserve its current homeostasis. In contrast, eliciting structural plasticity may require a more fundamental departure from homeostasis such that a new equilibrium state is reached, and then again maintained. Importing knowledge about critical periods in early ontogeny may prove to be useful in understanding and overcoming the inertia of the adult brain (Bavelier et al., 2010; Hensch, 2004; Morishita et al., 2010). In fact, it may turn out that much of the current efforts to elicit structural plasticity in the adult brain are similar to the attempt of speeding up your car with the brakes on. According to Bavelier et al. (2010), eliciting structural plasticity (in the sense of growth) in the adult mammalian brain may require selective resetting of the excitatory-inhibitory circuit balance to levels that resemble the balance that is present during critical periods in early ontogeny. Future research on humans inspired by this conceptual framework should seek to bridge the gap to animal models delineating the neurochemical preconditions for boosting plasticity in adulthood. New functional imaging methods that provide direct estimates of inhibitory and excitatory neural activity and related transmitter systems (Moran et al., 2011) may prove helpful in this regard.

To conclude, we hope that the present review has pointed out useful future directions and generated valuable theoretical propositions. Progress in the repertoire of non-invasive MR imaging techniques, in human brain data modeling, and in connecting human brain imaging to animal models nurture our confidence that these propositions can be turned into testable predictions.

\section{Conflicts of interest}

\section{None declared.}

\section{Acknowledgements}

Preparation of this review was funded by a grant from the Swedish Research Council (421-2010-1250) to ML. LB was supported by grants from the Swedish Research Council, Swedish Brain Power, an Alexander von Humboldt Research Award, and a donation from the af Jochnick Foundation. During the work on her dissertation, EW has been funded by the International Max Planck Research School "The Life Course: Evolutionary and Ontogenetic Dynamics” (LIFE).

\section{References}

Aguiar, A.S., Castro, A. A Moreira, E L Glaser, V., Santos, A.R S, Tasca, C. I, Latini, A Prediger, R.D.S., 2011. Short bouts of mild-intensity physical exercise improve spatial learning and memory in aging rats: involvement of hippocampal plasticity via AKT, CREB and BDNF signaling. Mechanisms of Ageing and Development $132,560-567$

Altman, J., Das, G.D., 1964. Autoradiographic examination of the effects of enriched environment on the rate of glial multiplication in the adult rat brain. Nature 204 , $1161-1163$.

Amunts, K., Schlaug, G., Jancke, L., Steinmetz, H., Schleicher, A., Dabringhaus, A., Zilles, K., 1997. Motor cortex and hand motor skills: structural compliance in the human brain. Human Brain Mapping 5, 206-215.

Anderson, B.J., 2011. Plasticity of gray matter volume: the cellular and synaptic plasticity that underlies volumetric change. Developmental Psychobiology 53, 456-465.

Anderson, B.J., Eckburg, P.B., Relucio, K.I., 2002. Alterations in the thickness of motor cortical subregions after motor-skill learning and exercise. Learning and Memory $9,1-9$. 
Anderson, B.J., Li, X., Alcantara, A.A., Isaacs, K.R., Black, J.E., Greenough, W.T., 1994. Glial hypertrophy is associated with synaptogenesis following motor-skill learning, but not with angiogenesis following exercise. Glia 11, 73-80.

Bavelier, D., Levi, D.M., Li, R.W., Dan, Y., Hensch, T.K., 2010. Removing brakes on adult brain plasticity: from molecular to behavioral interventions. Journal of Neuroscience 30, 14964-14971.

Beaulieu, C., 2002. The basis of anisotropic water diffusion in the nervous system a technical review. NMR in Biomedicine 15, 435-455.

Bennett, E.L., Diamond, M.C., Krech, D., Rosenzweig, M.R., 1964. Chemical and anatomical plasticity brain. Science 146, 610-619.

Bezzola, L., Merillat, S., Gaser, C., Jancke, L., 2011. Training-induced neural plasticity in golf novices. Journal of Neuroscience 31, 12444-12448.

Bhardwaj, R.D., Curtis, M.A., Spalding, K.L., Buchholz, B.A., Fink, D., Bjork-Eriksson, T., Nordborg, C., Gage, F.H., Druid, H., Eriksson, P.S., Frisen, J., 2006. Neocortical neurogenesis in humans is restricted to development. Proceedings of the National Academy of Sciences of the United States of America 103, 12564-12568.

Binder, D.K., Scharfman, H.E., 2004. Brain-derived neurotrophic factors. Growth Factors 22, 123-131.

Black, J.E., Isaacs, K.R., Anderson, B.J., Alcantara, A.A., Greenough, W.T., 1990. Learning causes synaptogenesis, whereas motor activity causes angiogenesis, in cerebellar cortex of adult rats. Proceedings of the National Academy of Sciences of the United States of America 87, 5568-5572.

Bloss, E.B., Janssen, W.G., Ohm, D.T., Yuk, F.J., Wadsworth, S., Saardi, K.M., McEwen, B.S., Morrison, J.H., 2011. Evidence for reduced experience-dependent dendritic spine plasticity in the aging prefrontal cortex. Journal of Neuroscience 31, 7831-7839.

Bookstein, F.L., 2001. "Voxel-based morphometry" should not be used with imperfectly registered images. Neuroimage 14, 1454-1462.

Boyke, J., Driemeyer, J., Gaser, C., Buchel, C., May, A., 2008. Training-induced brain structure changes in the elderly. Journal of Neuroscience 28, 7031-7035.

Briones, T.L., Klintsova, A.Y., Greenough, W.T., 2004. Stability of synaptic plasticity in the adult rat visual cortex induced by complex environment exposure. Brain Research 1018, 130-135

Callan, D.E., Jones, J.A., Callan, A.M., Akahane-Yamada, R., 2004. Phonetic perceptual identification by native- and second-language speakers differentially activates brain regions involved with acoustic phonetic processing and those involved with articulatory-auditory/orosensory internal models. Neuroimage 22, 1182-1194.

Caramanos, Z., Fonov, V.S., Francis, S.J., Narayanan, S., Pike, G.B., Collins, D.L., Arnold, D.L., 2010. Gradient distortions in MRI: characterizing and correcting for their effects on SIENA-generated measures of brain volume change. Neuroimage 49, 1601-1611.

Ceccarelli, A., Rocca, M.A., Pagani, E., Falini, A., Comi, G., Filippi, M., 2009. Cognitive learning is associated with gray matter changes in healthy human individuals: a tensor-based morphometry study. Neuroimage 48, 585-589.

Changeux, J.-P., Dehaene, S., 1989. Neuronal models of cognitive functions. Cognition 33, 64-109.

Clayton, N.S., Krebs, J.R., 1994. Hippocampal growth and attrition in birds affected by experience. Proceedings of the National Academy of Sciences of the United States of America 91, 7410-7414.

Colcombe, S., Erickson, K.I., Scalf, P., Kim, J.S., Prakash, R., McAuley, E., Elavsky, S., Marquez, D.X., Hu, L., Kramer, A., 2006. Aerobic exercise training increases brain volume in aging humans. Journal of Gerontology: Biological Sciences 61, $1166-1170$

Colom, R., Quiroga, M.Á., Solana, A.B., Burgaleta, M., Román, F.J., Privado, J., Escorial, S., Martínez, K., Álvarez-Linera, J., Alfayate, E., García, F., Lepage, C., Hernández-Tamames, J.A., Karama, S., 2012. Structural changes after videogame practice related to a brain network associated with intelligence. Intelligence 40 , 479-489.

Cowan, W.M., Fawcett, J.W., O’Leary, D.D., Stanfield, B.B., 1984. Regressive events in neurogenesis. Science 225, 1258-1265.

Davatzikos, C., 2004. Why voxel-based morphometric analysis should be used with great caution when characterizing group differences. Neuroimage 23, 17-20.

Davis, M.H., Gaskell, M.G., 2009. A complementary systems account of word learning: neural and behavioural evidence. Philosophical Transactions of the Royal Society B 364, 3773-3800.

Dayan, E., Cohen, L.G., 2011. Neuroplasticity subserving motor skill learning. Neuron $72,443-454$.

Demonet, J.F., Thierry, G., Cardebat, D., 2005. Renewal of the neurophysiology of language: functional neuroimaging. Physiological Reviews 85, 49-95.

Diaz, E., Pinto-Hamuy, T., Fernandez, V., 1994. Interhemispheric structural asymmetry induced by a lateralized reaching task in the rat motor cortex. European Journal of Neuroscience 6, 1235-1238

Draganski, B., Ashburner, J., Hutton, C., Kherif, F., Frackowiak, R.S., Helms, G., Weiskopf, N., 2011. Regional specificity of MRI contrast parameter changes in normal ageing revealed by voxel-based quantification (VBQ). Neuroimage 55, $1423-1434$

Draganski, B., Gaser, C., Busch, V., Schuierer, G., Bogdahn, U., May, A., 2004. Neuroplasticity: changes in grey matter induced by training. Nature 427, 311-312.

Draganski, B., Gaser, C., Kempermann, G., Kuhn, H.G., Winkler, J., Buchel, C., May, A., 2006. Temporal and spatial dynamics of brain structure changes during extensive learning. Journal of Neuroscience 26, 6314-6317.

Driemeyer, J., Boyke, J., Gaser, C., Buchel, C., May, A., 2008. Changes in gray matter induced by learning - revisited. PLoS One 3, e2669.

Dumitriu, D., Hao, J.D., Hara, Y., Kaufmann, J., Janssen, W.G.M., Lou, W., Rapp, P.R., Morrison, J.H., 2010. Selective changes in thin spine density and morphology in monkey prefrontal cortex correlate with aging-related cognitive impairment. Journal of Neuroscience 30, 7507-7515.

Duning, T., Kloska, S., Steinstrater, O., Kugel, H., Heindel, W., Knecht, S., 2005. Dehydration confounds the assessment of brain atrophy. Neurology 64, 548-550.

Dupret, D., Fabre, A., Dobrossy, M.D., Panatier, A., Rodriguez, J.J., Lamarque, S. Lemaire, V., Oliet, S.H.R., Piazza, P.V., Abrous, D.N., 2007. Spatial learning depends on both the addition and removal of new hippocampal neurons. PLoS Biology 5, 1683-1694.

Engvig, A., Fjell, A.M., Westlye, L.T., Moberget, T., Sundseth, O., Larsen, V.A., Walhovd, K.B., 2010. Effects of memory training on cortical thickness in the elderly. Neuroimage 52, 1667-1676.

Erickson, K.I., Voss, M.W., Prakash, R.S., Basak, C., Szabo, A., Chaddock, L., Kim, J.S., Heo, S., Alves, H., White, S.M., Wojcicki, T.R., Mailey, E., Vieira, V.J., Martin, S.A., Pence, B.D., Woods, J.A., McAuley, E., Kramer, A.F., 2011. Exercise training increases size of hippocampus and improves memory. Proceedings of the National Academy of Sciences of the United States of America 108 3017-3022

Fabel, K., Wolf, S.A., Ehninger, D., Babu, H., Leal-Galicia, P., Kempermann, G., 2009 Additive effects of physical exercise and environmental enrichment on adult hippocampal neurogenesis in mice. Frontiers in Neuroscience 3, 50.

Finkel, D., Andel, R., Gatz, M., Pedersen, N.L., 2009. The role of occupational complexity in trajectories of cognitive aging before and after retirement. Psychology and Aging 24, 563-573.

Fischl, B., Dale, A.M., 2000. Measuring the thickness of the human cerebral cortex from magnetic resonance images. Proceedings of the National Academy of Sciences of the United States of America 97, 11050-11055.

Fjell, A.M., Walhovd, K.B., Fennema-Notestine, C., McEvoy, L.K., Hagler, D.J., Holland, D., Brewer, J.B., Dale, A.M., 2009. One-year brain atrophy evident in healthy aging. Journal of Neuroscience 29, 15223-15231.

Fratiglioni, L., Paillard-Borg, S., Winblad, B., 2004. An active and socially integrated lifestyle in late life might protect against dementia. The Lancet Neurology 3 343-353.

Fu, M., Zuo, Y., 2011. Experience-dependent structural plasticity in the cortex. Trends in Neuroscience 34, 177-187.

Gaser, C., Schlaug, G., 2003. Brain structures differ between musicians and non-musicians. Journal of Neuroscience 23, 9240-9245.

Gomez-Pinilla, F., So, V., Kesslak, J.P., 1998. Spatial learning and physica activity contribute to the induction of fibroblast growth factor: neural substrates for increased cognition associated with exercise. Neuroscience 85, 53-61.

Gould, E., Beylin, A., Tanapat, P., Reeves, A., Shors, T.J., 1999. Learning enhances adult neurogenesis in the hippocampal formation. Nature Neuroscience 2, 260-265.

Granert, O., Peller, M., Gaser, C., Groppa, S., Hallett, M., Knutzen, A., Deuschl, G., Zeuner, K.E., Siebner, H.R., 2011. Manual activity shapes structure and function in contralateral human motor hand area. Neuroimage 54, 32-41.

Green, E.J., Greenough, W.T., Schlumpf, B.E., 1983. Effects of complex or isolated environments on cortical dendrites of middle-aged rats. Brain Research 264 233-240.

Greenough, W.T., Mcdonald, J.W., Parnisari, R.M., Camel, J.E., 1986. Environmental-conditions modulate degeneration and new dendrite growth in cerebellum of senescent rats. Brain Research 380, 136-143.

Greenough, W.T., Volkmar, F.R., Juraska, J.M., 1973. Effects of rearing complexity on dendritic branching in frontolateral and temporal cortex of the rat. Experimenta Neurology 41, 371-378

Grossman, A.W., Churchill, J.D., Bates, K.E., Kleim, J.A., Greenough, W.T., 2002. A brain adaptation view of plasticity: is synaptic plasticity an overly limited concept? Progress in Brain Research 138, 91-108.

Grutzendler, J., Kasthuri, N., Gan, W.B., 2002. Long-term dendritic spine stability in the adult cortex. Nature 420, 812-816.

Gryga, M., Taubert, M., Dukart, J., Vollmann, H., Conde, V., Sehm, B., Villringer, A. Ragert, P., 2012. Bidirectional gray matter changes after complex motor skil learning. Frontiers in Systems Neuroscience 6, 37.

Haier, R.J., Karama, S., Leyba, L., Jung, R.E., 2009. MRI assessment of cortical thickness and functional activity changes in adolescent girls following three months of practice on a visual-spatial task. BMC Research Notes 2, 174.

Hamzei, F., Glauche, V., Schwarzwald, R., May, A., 2012. Dynamic gray matter changes within cortex and striatum after short motor skill training are associated with their increased functional interaction. Neuroimage 59, 3364-3372.

Hebb, D.O., 1949. The Organization of Behavior. Wiley, New York.

Hensch, T.K., 2004. Critical period regulation. Annual Review of Neuroscience 27, 549-579.

Hertzog, C., Kramer, A.F., Wilson, R.S., Lindenberger, U., 2009. Enrichment effects on adults cognitive development. Psychological Science in the Public Interest 9 $1-65$.

Hickok, G., Poeppel, D., 2007. Opinion - the cortical organization of speech processing. Nature Reviews Neuroscience 8, 393-402.

Holtmaat, A., Svoboda, K., 2009. Experience-dependent structural synaptic plasticity in the mammalian brain. Nature Reviews Neuroscience 10, 647-658.

Holtmaat, A.J.G.D., Trachtenberg, J.T., Wilbrecht, L., Shepherd, G.M., Zhang, X.Q. Knott, G.W., Svoboda, K., 2005. Transient and persistent dendritic spines in the neocortex in vivo. Neuron 45, 279-291.

Hölzel, B.K., Carmody, J., Vangel, M., Congleton, C., Yerramsetti, S.M., Gard, T., Lazar, S.W., 2011. Mindfulness practice leads to increases in regional brain gray matter density. Psychiatry Research 191, 36-43. 
Hutton, C., Draganski, B., Ashburner, J., Weiskopf, N., 2009. A comparison between voxel-based cortical thickness and voxel-based morphometry in normal aging. Neuroimage 48, 371-380.

Ilg, R., Wohlschlager, A.M., Gaser, C., Liebau, Y., Dauner, R., Woller, A., Zimmer, C., Zihl, J., Muhlau, M., 2008. Gray matter increase induced by practice correlates with task-specific activation: a combined functional and morphometric magnetic resonance imaging study. Journal of Neuroscience 28, 4210-4215.

Johansen-Berg, H., Baptista, C.S., Thomas, A.G., 2012. Human structural plasticity at record speed. Neuron 73, 1058-1060.

Jones, T.A., Klintsova, A.Y., Kilman, V.L., Sirevaag, A.M., Greenough, W.T., 1997. Induction of multiple synapses by experience in the visual cortex of adult rats. Neurobiology of Learning and Memory 68, 13-20.

Jovicich, J., Czanner, S., Greve, D., Haley, E., van der Kouwe, A., Gollub, R., Kennedy, D. Schmitt, F., Brown, G., Macfall, J., Fischl, B., Dale, A., 2006. Reliability in multi-site structural MRI studies: effects of gradient non-linearity correction on phantom and human data. Neuroimage 30, 436-443.

Juraska, J.M., Fitch, J.M., Washburne, D.L., 1989. The dendritic morphology of pyramidal neurons in the rat hippocampal CA3 area. II. Effects of gender and the environment. Brain Research 479, 115-119.

Kasai, H., Matsuzaki, M., Noguchi, J., Yasumatsu, N., Nakahara, H., 2003. Structure-stability-function relationships of dendritic spines. Trends in Neuroscience 26, 360-368.

Kelly, A.M., Garavan, H., 2005. Human functional neuroimaging of brain changes associated with practice. Cerebral Cortex 15, 1089-1102.

Kempermann, G., 1997. More hippocamal neurons in adult mice living in an enriched environment. Nature 386, 493-495.

Kempermann, G., 2008. The neurogenic reserve hypothesis: what is adult hippocam pal neurogenesis good for? Trends in Neuroscience 31, 163-169.

Kempermann, G., Gast, D., Gage, F.H., 2002. Neuroplasticity in old age: sustained fivefold induction of hippocampal neurogenesis by long-term environmental enrichment. Annals of Neurology 52, 135-143.

Kempermann, G., Kuhn, H.G., Gage, F.H., 1998. Experience-induced neurogenesis in the senescent dentate gyrus. Journal of Neuroscience 18, 3206-3212.

Kempton, M.J., Ettinger, U., Schmechtig, A., Winter, E.M., Smith, L., McMorris T., Wilkinson, I.D., Williams, S.C., Smith, M.S., 2009. Effects of acute dehydration on brain morphology in healthy humans. Human Brain Mapping 30 291-298.

Kim, P., Leckman, J.F., Mayes, L.C., Feldman, R., Wang, X., Swain, J.E., 2010. The plasticity of human maternal brain: longitudinal changes in brain anatomy during the early postpartum period. Behavioral Neuroscience 124, 695-700.

Kirkwood, T.B., 2005. Understanding the odd science of aging. Cell 120, 437-447.

Kleim, J.A., Hogg, T.M., VandenBerg, P.M., Cooper, N.R., Bruneau, R., Remple, M., 2004 Cortical synaptogenesis and motor map reorganization occur during late, but not early, phase of motor skill learning. Journal of Neuroscience 24, 628-633.

Kleim, J.A., Lussnig, E., Schwarz, E.R., Comery, T.A., Greenough, W.T., 1996. Synaptogenesis and Fos expression in the motor cortex of the adult rat after motor skil learning. Journal of Neuroscience 16, 4529-4535.

Kleim, J.A., Swain, R.A., Armstrong, K.A., Napper, R.M., Jones, T.A., Greenough, W.T. 1998. Selective synaptic plasticity within the cerebellar cortex following complex motor skill learning. Neurobiology of Learning and Memory 69, 274-289.

Kolb, B., Cioe, J., Comeau, W., 2008. Contrasting effects of motor and visual spatial learning tasks on dendritic arborization and spine density in rats. Neurobiology of Learning and Memory 90, 295-300.

Kolb, B., Forgie, M., Gibb, R., Gorny, G., Rowntree, S., 1998. Age, experience and the changing brain. Neuroscience and Biobehavioral Reviews 22, 143-159.

Kozorovitskiy, Y., Gross, C.G., Kopil, C., Battaglia, L., McBreen, M., Stranahan, A.M. Gould, E., 2005. Experience induces structural and biochemical changes in the adult primate brain. Proceedings of the National Academy of Sciences of the United States of America 102, 17478-17482.

Kriegeskorte, N., Simmons, W.K., Bellgowan, P.S., Baker, C.I., 2009. Circular analysis in systems neuroscience: the dangers of double dipping. Nature Neuroscience $12,535-540$

Kwok, V., Niu, Z.D., Kay, P., Zhou, K., Mo, L., Jin, Z., So, K.F., Tan, L.H., 2011. Learning new color names produces rapid increase in gray matter in the intact adult human cortex. Proceedings of the National Academy of Sciences of the United States of America 108, 6686-6688.

Landi, S.M., Baguear, F., Della-Maggiore, V., 2011. One week of motor adaptation induces structural changes in primary motor cortex that predict long-term memory one year later. Journal of Neuroscience 31, 11808-11813.

Langer, N., Hanggi, J., Muller, N.A., Simmen, H.P., Jancke, L., 2012. Effects of limb immobilization on brain plasticity. Neurology 78, 182-188.

Le Bihan, D., 2007. The 'wet mind': water and functional neuroimaging. Physics in Medicine and Biology 52, R57-R90.

Lerch, J.P., Yiu, A.P., Martinez-Canabal, A., Pekar, T., Bohbot, V.D., Frankland, P.W., Henkelman, R.M., Josselyn, S.A., Sled, J.G., 2011. Maze training in mice induces MRI-detectable brain shape changes specific to the type of learning. Neuroimage $54,2086-2095$

Lindenberger, U., Burzynska, A.Z., Nagel, I., 2012. Heterogeneity in frontal lobe aging, in: Stuss, D.T., Knight, R.T. (Eds.), Principles of Frontal Lobe Functions, 2nd ed. Oxford University Press, New York.

Lindenberger, U., Li, S.C., Bäckman, L. 2006. Delineating brain-behavior mappings across the lifespan: substantive and methodological advances in developmental neuroscience. Neuroscience and Biobehavioral Reviews 30, 713-717.

Lövdén, M., Bäckman, L., Lindenberger, U., Schaefer, S., Schmiedek, F., 2010. A theoretical framework for the study of adult cognitive plasticity. Psychological Bulletin 136, 659-676
Lövdén, M., Ghisletta, P., Lindenberger, U., 2005. Social participation attenuates decline in perceptual speed in old and very old age. Psychology and Aging 20, 423-434.

Lövdén, M., Schaefer, S., Noack, H., Bodammer, N.C., Kuhn, S., Heinze, H.J., Duzel, E., Bäckman, L., Lindenberger, U., 2012. Spatial navigation training protects the hippocampus against age-related changes during early and late adulthood. Neurobiology of Aging 33, 620 e629-620 e622.

Lövdén, M., Schaefer, S., Noack, H., Kanowski, M., Kaufmann, J., Tempelmann, C. Bodammer, N.C., Kuhn, S., Heinze, H.J., Lindenberger, U., Duzel, E., Bäckman, L. 2011. Performance-related increases in hippocampal N-acetylaspartate (NAA) induced by spatial navigation training are restricted to BDNF Val homozygotes. Cerebral Cortex 21, 1435-1442.

Lu, B., 2003. BDNF and activity-dependent synaptic modulation. Learning and Memory $10,86-98$.

Luo, L., O’Leary, D.D., 2005. Axon retraction and degeneration in development and disease. Annual Review of Neuroscience 28, 127-156.

Maguire, E.A., Gadian, D.G., Johnsrude, I.S., Good, C.D., Ashburner, J., Frackowiak, R.S. Frith, C.D., 2000. Navigation-related structural change in the hippocampi of taxi drivers. Proceedings of the National Academy of Sciences of the United States of America 97, 4398-4403.

Markham, J.A., Greenough, W.T., 2004. Experience-driven brain plasticity: beyond the synapse. Neuron Glia Biology 1, 351-363.

May, A., Gaser, C., 2012. Response to Thomas and Baker: the structural adaptations of the brain to training. Trends in Cognitive Sciences 19, 97-98.

Mårtensson, J., Eriksson, J., Bodammer, N.C., Lindgren, M., Johansson, M., Nyberg, L. Lövdén, M., 2012. Growth of language-related brain areas after foreign language learning. Neuroimage 63, 240-244.

May, A., 2011. Experience-dependent structural plasticity in the adult human brain. Trends in Cognitive Sciences 15, 475-482.

Meister, I.G., Wilson, S.M., Deblieck, C., Wu, A.D., Lacoboni, M., 2007. The essential role of premotor cortex in speech perception. Current Biology 17, 1692-1696.

Moffett, J.R., Ross, B., Arun, P., Madhavarao, C.N., Namboodiri, A.M.A., 2007. $\mathrm{N}$-acetylaspartate in the CNS: from neurodiagnostics to neurobiology. Progress in Neurobiology 81, 89-131.

Molina-Luna, K., Hertler, B., Buitrago, M.M., Luft, A.R., 2008. Motor learning transiently change cortical somatotopy. Neuroimage 40, 1748-1754

Moran, R.J., Symmonds, M., Stephan, K.E., Friston, K.J., Dolan, R.J., 2011. An in vivo assay of synaptic function mediating human cognition. Current Biology 21 1320-1325

Morishita, H., Miwa, J.M., Heintz, N., Hensch, T.K., 2010. Lynx1, a cholinergic brake, limits plasticity in adult visual cortex. Science 330, 1238-1240.

Moser, M.B., Trommald, M., Andersen, P., 1994. An increase in dendritic spine density on hippocampal CA1 pyramidal cells following spatial learning in adult rats suggests the formation of new synapses. Proceedings of the National Academy of Sciences of the United States of America 91, 12673-12675.

Nieuwenhuis, S., Forstmann, B.U., Wagenmakers, E.J., 2011. Erroneous analyses of interactions in neuroscience: a problem of significance. Nature Neuroscience 14 1105-1107

Nithianantharajah, J., Hannan, A.J., 2009. The neurobiology of brain and cognitive reserve: mental and physical activity as modulators of brain disorders. Progress in Neurobiology 89, 369-382.

Nyberg, L., Lövdén, M., Riklund, K., Lindenberger, U., Bäckman, L., 2012. Memory aging and brain maintenance. Trends in Cognitive Sciences 16, 292-305.

Palmer, T.D., Willhoite, A.R., Gage, F.H., 2000. Vascular niche for adult hippocampal neurogenesis. Journal of Comparative Neurology 425, 479-494

Poo, M., 2001. Neurotrophins as synaptic modulators. Nature Reviews Neuroscience $2,24-32$.

Price, C.J., 2010. The anatomy of language: a review of $100 \mathrm{fMRI}$ studies published in 2009. Annals of the New York Academy of Sciences 1191, 62-88

Quallo, M.M., Price, C.J., Ueno, K., Asamizuya, T., Cheng, K., Lemon, R.N., Iriki, A., 2009. Gray and white matter changes associated with tool-use learning in macaque monkeys. Proceedings of the National Academy of Sciences of the United States of America 106, 18379-18384.

Raz, N., Lindenberger, U., Rodrigue, K.M., Kennedy, K.M., Head, D., Williamson, A Dahle, C., Gerstorf, D., Acker, J.D., 2005. Regional brain changes in aging healthy adults: general trends, individual differences and modifiers. Cerebral Cortex 15 $1676-1689$.

Reed, A., Riley, J., Carraway, R., Carrasco, A., Perez, C., Jakkamsetti, V., Kilgard, M.P., 2011. Cortical map plasticity improves learning but is not necessary for improved performance. Neuron 70, 121-131.

Rosenzweig, M.L., Bennett, E.L., Diamond, M.C., 1972. Brain changes in response to experience. Scientific American 22, 22-29.

Rosenzweig, M.R., Bennett, E.L., 1996. Psychobiology of plasticity: effects of training and experience on brain and behavior. Behavioural Brain Research 78, 57-65.

Rosenzweig, M.R., Bennett, E.L., Krech, D., 1964. Cerebral effects of environmental complexity and training among adult rats. Journal of Comparative and Physiological Psychology 57, 438-439.

Rosenzweig, M.R., Krech, D., Bennett, E.L., Zolman, J.F., 1962. Variation in environmental complexity and brain measures. Journal of Comparative and Physiological Psychology 55, 1092-1095

Rosenzweig, M.R., Love, W., Bennett, E.L., 1968. Effects of a few hours a day of enriched enxpereince on brain chemistry and brain weights. Physiology and Behavior 3, 819-825.

Sagi, Y., Tavor, I., Hofstetter, S., Tzur-Moryosef, S., Blumenfeld-Katzir, T., Assaf, Y., 2012. Learning in the fast lane: new insights into neuroplasticity. Neuron 73 , 1195-1203. 
Schlaug, G., Jancke, L., Huang, Y.X., Steinmetz, H., 1995. In-vivo evidence of structural brain asymmetry in musicians. Science 267, 699-701.

Schmidt-Wilcke, T., Rosengarth, K., Luerding, R., Bogdahn, U., Greenlee, M.W., 2010. Distinct patterns of functional and structural neuroplasticity associated with learning Morse code. Neuroimage 51, 1234-1241.

Scholz, J., Klein, M.C., Behrens, T.E., Johansen-Berg, H., 2009. Training induces changes in white-matter architecture. Nature Neuroscience 12, 1370-1371.

Senjem, M.L., Gunter, J.L., Shiung, M.M., Petersen, R.C., JackJr., C.R., 2005. Comparison of different methodological implementations of voxel-based morphometry in neurodegenerative disease. Neuroimage 26, 600-608.

Snyder, J.S., Cameron, H.A., 2012. Could adult hippocampal neurogenesis be relevant for human behavior? Behavioural Brain Research 227, 384-390.

Stein, M., Federspiel, A., Koenig, T., Wirth, M., Strik, W., Wiest, R., Brandeis, D., Dierks, T., 2010. Structural plasticity in the language system related to increased second language proficiency. Cortex 48, 458-465.

Stern, Y., 2009. Cognitive reserve. Neuropsychologia 47, 2015-2028.

Sykova, E., Nicholson, C., 2008. Diffusion in brain extracellular space. Physiological Reviews 88, 1277-1340.

Takeuchi, H., Taki, Y., Sassa, Y., Hashizume, H., Sekiguchi, A., Fukushima, A., Kawashima, R., 2011. Working memory training using mental calculation impacts regional gray matter of the frontal and parietal regions. PLoS One 6, e23175.

Tang, Y.-Y., Lu, Q., Geng, X., Stein, E.A., Yang, Y., Posner, M.I., 2010. Short-term meditation induces white matter changes in the anterior cingulate. Proceedings of the National Academy of Sciences of the United States of America 107,15649-15652.

Tata, D.A., Marciano, V.A., Anderson, B.J., 2006. Synapse loss from chronically elevated glucocorticoids: relationship to neuropil volume and cell number in hippocampal area CA3. Journal of Comparative Neurology 498, 363-374.

Taubert, M., Draganski, B., Anwander, A., Muller, K., Horstmann, A., Villringer, A., Ragert, P., 2010. Dynamic properties of human brain structure: learning-related changes in cortical areas and associated fiber connections. Journal of Neuroscience 30, 11670-11677.

Teutsch, S., Herken, W., Bingel, U., Schoell, E., May, A., 2008. Changes in brain gray matter due to repetitive painful stimulation. Neuroimage 42, 845-849.

Thomas, A.G., Marrett, S., Saad, Z.S., Ruff, D.A., Martin, A., Bandettini, P.A., 2009. Functional but not structural changes associated with learning: an exploration of longitudinal voxel-based morphometry (VBM). Neuroimage 48, 117-125.
Thomas, C., Baker, C.I., in press. Teaching an adult brain new tricks: A critical review of evidence for training-dependent structural plasticity in humans. Neuroimage. Tost, H., Braus, D.F., Hakimi, S., Ruf, M., Vollmert, C., Hohn, F., Meyer-Lindenberg A., 2010. Acute D2 receptor blockade induces rapid, reversible remodeling in human cortical-striatal circuits. Nature Neuroscience 13 , 920-922.

Tronel, S., Fabre, A., Charrier, V., Oliet, S.H., Gage, F.H., Abrous, D.N., 2010. Spatial learning sculpts the dendritic arbor of adult-born hippocampal neurons. Proceedings of the National Academy of Sciences of the United States of America 107, 7963-7968.

van Praag, H., Kempermann, G., Gage, F.H., 2000. Neural consequences of environmental enrichment. Nature Reviews Neuroscience 1, 191-198.

Volkmar, F.R., Greenough, W.T., 1972. Rearing complexity affects branching of dendrites in the visual cortex of the rat. Science 176, 1445-1447.

Wagner, A.P., Schmoll, H., Badan, I., Platt, D., Kessler, C., 2000. Brain plasticity: to what extent do aged animals retain the capacity to coordinate gene activity in response to acute challenges. Experimental Gerontology 35, 1211-1227.

Waimey, K.E., Cheng, H.J., 2006. Axon pruning and synaptic development: how are they per-plexin? Neuroscientist 12, 398-409.

Wenger, E., Schaefer, S., Noack, H., Kuhn, S., Mårtensson, J., Heinze, H.J., Duzel, E., Bäckman, L., Lindenberger, U., Lövdén, M., 2012. Cortical thickness changes following spatial navigation training in adulthood and aging. Neuroimage 59, 3389-3397.

Withers, G.S., Greenough, W.T., 1989. Reach training selectively alters dendritic branching in subpopulations of layer II-III pyramids in rat motor-somatosensory forelimb cortex. Neuropsychologia 27, 61-69.

Woollett, K., Maguire, E.A., 2011. Acquiring "the Knowledge" of London's layout drives structural brain changes. Current biology: CB 21, 2109-2114.

Xu, T., Yu, X., Perlik, A.J., Tobin, W.F., Zweig, J.A., Tennant, K., Jones, T., Zuo, Y., 2009 Rapid formation and selective stabilization of synapses for enduring motor memories. Nature 462, 915-919.

Yang, G., Pan, F., Gan, W.B., 2009. Stably maintained dendritic spines are associated with lifelong memories. Nature 462, 920-924.

Zatorre, R.J., Fields, R.D., Johansen-Berg, H., 2012. Plasticity in gray and white: neuroimaging changes in brain structure during learning. Nature Neuroscience 15 528-536.

Zhao, C., Deng, W., Gage, F.H., 2008. Mechanisms and functional implications of adult neurogenesis. Cell 132, 645-660. 